\title{
THE DETERMINATION OF THE IMAGINARY ABELIAN NUMBER FIELDS WITH CLASS NUMBER ONE
}

\author{
KEN YAMAMURA
}

\begin{abstract}
In this paper, we determine all the imaginary abelian number fields with class number one. There exist exactly 172 imaginary abelian number fields with class number one. The maximal conductor of these fields is $10921=67$. 163 , which is the conductor of the biquadratic number field $Q(\sqrt{-67}, \sqrt{-163})$.
\end{abstract}

\section{INTRODUCTION}

Uchida proved that there exist only finitely many imaginary abelian number fields with class number one [28], and gave the value $2 \times 10^{10}$ as an upper bound for the conductors of such fields [29]. Several authors determined such fields of some types, but not all of them have been determined yet. (Masley determined the cyclotomic number fields with class number one [21], and Uchida determined such fields having a power-of-two degree [30].) ${ }^{1}$ In this paper, we shall determine all the imaginary abelian number fields with class number one.

Theorem. There exist exactly 172 imaginary abelian number fields with class number one as given in the table at the end of the paper. Among them, 29 fields are cyclotomic, 49 fields are cyclic, and 88 fields are maximal with respect to inclusion. The maximal conductor of these fields is $10921=67 \cdot 163$, which is the conductor of the biquadratic number field $\mathbf{Q}(\sqrt{-67}, \sqrt{-163})$.

We note that among the 172 fields, at least 132 fields (at least 155 fields if the Generalized Riemann Hypothesis is true) do not have any nontrivial unramified extension. (We describe the details in the Appendix.) One of the main motivations for this work is to construct many fields with this property.

We now sketch the method of proof. The basic idea is due to Uchida. (See $[29,30]$.) Throughout this paper, let $K$ be an imaginary abelian number field with class number $h(K)$. When $h(K)=1$, the genus number ${ }^{2}$ of $K$ is one, which is equivalent to saying that the group $X$ of Dirichlet characters associated with $K$ is a direct product of subgroups generated by a character of prime power conductor:

$$
X=\left\langle\chi_{1}\right\rangle \times \cdots \times\left\langle\chi_{r}\right\rangle,
$$

Received by the editor March 17, 1992 and, in revised form, September 14, 1992 and March 23, 1993.

1991 Mathematics Subject Classification. Primary 11R20, 11Y40.

Key words and phrases. Imaginary abelian number fields, class number, characters.

${ }^{1}$ Recently, Louboutin determined the imaginary abelian sextic number fields with class number one [12]. The author learned of his result after completion of this work.

${ }^{2}$ The extension degree $\left[K^{*}, K\right]$, where $K^{*}$ is the genus field of $K$. 
where the conductor $f_{\chi_{i}}$ of each $\chi_{i}$ is a prime power. (In the following, we shall take $\chi_{i}$ so that the number $r$ of them is minimal (for details, see $\S 3$ ).) The key idea which facilitates the determination is:

(*) For any subfield $F$ of $K$ with genus number one, $h(F)$ divides $h(K)$. Because of this, we need consider only $K$ with genus number one such that

(\#) any (proper) subfield with genus number one has class number one.

Among such $K$, we first determine $K$ with $h^{-}(K)=1$, and then check whether or not $h^{+}(K)=1$. Here, $h^{-}(K)$ (resp. $h^{+}(K)$ ) denotes the relative class number of $K$ (resp. the class number of the maximal real subfield $K^{+}$of $K$ ). $\left(h(K)=h^{-}(K) h^{+}(K)\right.$.) By $(*)$, in most cases we can immediately restrict $K$ to be considered by using data on fields with smaller character group. Thus, it is reasonable to start with small character group. Let $L$ be the subfield of $K$ corresponding to the 2-Sylow subgroup of $X$. Then we easily see that the genus number of $L$ is one. Therefore, if $h(K)=1$, then $L$ is also an imaginary abelian number field with class number one, which is noted by Uchida [30]. Since $K$ is imaginary, at least one $\chi_{i}$ is odd. We may, and shall, always take $\chi_{1}$ to be an odd generator of minimal order. We first treat the case where the conductor of $K$ is a power of a prime number $p$. In this case, since the possibilities of $L$ are known, the possibilities of the values of $p$ are known, and the determination is easy. Next, we treat the case where $r=2$, and ord $\chi_{1}$ is a power of two and ord $\chi_{2}$ is a power of two or an odd prime. In this case, we need the help of a computer for the calculation of relative class numbers of many fields. The other cases are easily treated by using the data in the above two basic cases. (For details, see $\S 3$ )

In order to reduce the amount of computer calculation, we need a good lower bound for $h^{-}(K)$. This problem is reduced to that of getting a good upper bound for $|L(1, \chi)|$ for nontrivial even Dirichlet characters $\chi \in X$ by using Uchida's estimation for $h^{-}(K)$ ([30, Proposition 1]) and several results on real zeros of the Dedekind zeta function (Low [15], Rosser [26] and Chowla [2]). Examining Moser and Payan's proof in [22, §3], we get

Proposition. Let $\chi$ be a primitive nontrivial even Dirichlet character of conductor f. Then

$$
|L(1, \chi)|<\frac{1}{2} \log f+\gamma-\frac{1}{2}
$$

where $\gamma$ is the Euler constant.

Now we describe a proof of Proposition. Moser and Payan proved it in [22] in the case where $f$ is a prime number. But their proof does not depend on the primeness of $f$. (Cf. Hua's proof of a weaker result for quadratic characters [9].) There is the restriction $f>100$ in [22], and the proof contains slight mistakes. Correcting them, $f>100$ can be read as $f \geqq 30$. Calculating $|L(1, \chi)|$ approximately when $f<30$, we conclude that we can remove also the restriction on the size of $f$.

This Proposition enables us to execute all the calculations on the personal computer NEC PC-9801. 
The largest part of the check $h^{+}(K)=1$ is done by using results of Masley [20] and Mäki [16].

\section{LOWER ESTIMATE FOR $h^{-}(K)$}

As described in the Introduction, we use a computer for the calculation of $h^{-}(K)$ for many $K$. In this section we describe a lower estimate for $h^{-}(K)$. The analytic relative class number formula is

$$
h^{-}(K)=\frac{Q_{K} w_{K}}{(2 \pi)^{n / 2}} \sqrt{\frac{D_{K}}{D_{K^{+}}}} \prod_{\chi: \text { odd }} L(1, \chi),
$$

where

$$
\begin{aligned}
& Q_{K}=\left[E_{K}: W_{K} E_{K^{+}}\right]: \text {the unit index, } \\
& E_{K}: \text { the group of units in } K, \\
& W_{K}: \text { the group of roots of unity in } K, \\
& w_{K}=\# W_{K}: \text { the number of roots of unity in } K, \\
& n=[K: \mathbf{Q}]: \text { the degree of } K, \\
& D_{K}: \text { the absolute value of the discriminant of } K,
\end{aligned}
$$

and $\chi$ runs over all odd characters in $X$. For simplicity, we denote by $L_{1}(s)$ (resp. $L_{0}(s)$ ) the product of $L$-functions for all odd (resp. nontrivial even) characters in $X$. Uchida gave a lower bound for $L_{1}(1)$ by using the values of $L_{0}(s)$ :

Proposition U1 (Uchida [30, Proposition 1]). Let $K$ be an imaginary abelian number field of degree $\geqq 4$ such that $L_{1}(s)$ has no exceptional zero. Then

$$
L_{1}(1)^{-1} \leqq 9.3 L_{0}\left(s_{0}\right) \log D_{K},
$$

or

$$
L_{1}(1)^{-1} \leqq 9.3 L_{0}(1) \log D_{K},
$$

according as the Dedekind zeta function $\zeta_{K}(s)$ of $K$ has an exceptional zero or not. Here,

$$
s_{0}=1+\frac{1}{1.2 \log D_{K}},
$$

and a zero $\rho$ of $\zeta_{K}(s)$ is called exceptional if it satisfies the inequality

$$
\Re \frac{1}{s_{0}-\rho}>0.85 \log D_{K} \cdot^{3}
$$

We note that the inequality

$$
0.85 \log D_{K}>\frac{1}{2} \sum_{\rho} \Re \frac{1}{s_{0}-\rho}
$$

always holds, where $\rho$ runs over all zeros of $\zeta_{K}(s)$ such that $0<\Re \rho<1$ (see $\left[30\right.$, p.153]). If $\rho$ is a zero of $\zeta_{K}(s)$, then its complex conjugate $\bar{\rho}$ is also a

\footnotetext{
${ }^{3}$ The definition of exceptional zero in [30] should be rewritten like this.
} 
zero of $\zeta_{K}(s)$. Therefore, if $\zeta_{K}(s)$ has an exceptional zero, then it is a real simple zero. Thus, an exceptional zero is a real zero $\rho$ of $\zeta_{K}(s)$ such that

$$
\rho>1-\frac{35}{102 \log D_{K}} .
$$

Any positive real simple zero of $\zeta_{K}(s)$ is a zero of $L(s, \chi)$ associated with a quadratic subfield of $K$. For, if $\chi$ is a nontrivial nonquadratic character belonging to $X$, then $\bar{\chi}$ belongs to $X$ and $\chi \neq \bar{\chi}$, and therefore, we have the factorization

$$
\zeta_{K}(s)=\zeta(s) \prod_{\chi: \text { quadratic }} L(s, \chi) \prod_{\substack{\{\chi, \bar{\chi}\} \\ \chi: \text { nonquadratic }}} L(s, \chi) \overline{L(s, \chi)}
$$

for $s>0$, because $L(s, \bar{\chi})=\overline{L(s, \chi)}$ for real $s$. Thus, the main difficulty of the (class number) problem lies with quadratic number fields. (See also [8, 27].) This work owes much to Uchida's results. But, Uchida needed Baker and Stark's results (the determination of the imaginary quadratic number fields with class number one and two).

For real zeros of $L(s, \chi)$ associated with a quadratic field, the following is known:

Theorem $\mathbf{R}$ (Rosser [26]). Let $\chi$ be a nontrivial real Dirichlet character of conductor $f$. If $f \leqq 227$, then $L(s, \chi)$ has no positive real zero.

Theorem L (Low [15]). Let $\chi$ be an odd quadratic Dirichlet character of conductor $f$. If $f \leqq 593000$, then $L(s, \chi)>0$ for $s>0$.

It has long been conjectured that for any quadratic character $\chi$, one has $L(s, \chi)>0$ for $s>0$. But this conjecture has not yet been settled. Chowla gave a necessary condition for $L(s, \chi)>0 \quad(s>0)$, which we can easily check by a computer for some $\chi$ of small conductors:

Theorem C (Chowla [2]). Let $\chi$ be a quadratic Dirichlet character and $S_{m}(x)$ the mth character sum:

$$
S_{1}(x)=\sum_{n=1}^{x} \chi(n), \quad S_{m}(x)=\sum_{n=1}^{x} S_{m-1}(n) \quad(m \geqq 2) .
$$

If there exists a positive integer $m$ such that $S_{m}(x) \geqq 0$ for all $x \geqq 1$, then $L(s, \chi)>0$ for $s>0$.

In addition to Theorems $\mathrm{R}$ and $\mathrm{L}$, using this, ${ }^{4}$ we checked by a computer that for any imaginary abelian number field $L$ of power-of-two degree with class number one, neither $L_{1}(s)$ nor $\zeta_{L}(s)$ has an exceptional zero. Therefore, for all $K$ we consider $L_{1}(1)^{-1} \leqq 9.3 L_{0}(1) \log D_{K}$ by Proposition U1. Thus,

\footnotetext{
${ }^{4}$ Heilbronn [7] proved there exist infinitely many quadratic characters $\chi$ such that for any $m \geqq 1, S_{m}(x)<0$ for some $x$. For this reason, some modified versions of Theorem $\mathrm{C}$ were considered by several authors (see $[26,3,4,5]$ ). But for our check, Theorem $C$ is sufficient.
} 
we can estimate $h^{-}(K)$ from below by estimating $|L(1, \chi)|$ from above for nontrivial even $\chi \in X$. Therefore, by the conductor-discriminant formula and Proposition, we have

$$
h^{-}(K)>\frac{Q_{K} w_{K} \sqrt{\prod_{\chi: \text { odd }} f_{\chi}}}{(2 \pi)^{n / 29.3}\left\{\prod_{\substack{\chi \text { even } \\ \chi \neq 1}}\left(\frac{1}{2} \log f_{\chi}+\gamma-\frac{1}{2}\right)\right\}\left(\sum_{\chi} \log f_{\chi}\right)},
$$

where $f_{\chi}$ is the conductor of $\chi \cdot{ }^{5}$ Before the actual calculation of $h^{-}(K)$, we use this to decide the amount of calculation. For actual calculation, we use the arithmetic formula

$$
h^{-}(K)=Q_{K} w_{K} \prod_{\chi: \text { odd }}\left(-\frac{1}{2} B_{1, \chi}\right), \quad B_{1, \chi}=\frac{1}{f_{\chi}} \sum_{a=1}^{f_{\chi}} \chi(a) a,
$$

and the formula for the absolute norm of $-\frac{1}{2} B_{1, \chi}$ for $\chi$ whose conductor is not a prime power, given in $[6, \S 28]$.

For the unit index $Q_{K}$, Uchida gave a simple criterion:

Proposition U2 (Uchida [29, Proposition 3]). Let $K$ be an imaginary abelian number field with genus number one so that $K$ is the composite field $K_{1} \cdots K_{t}$ of its subfields $K_{i}$ of prime power conductors which are pairwise relatively prime. Then the unit index $Q_{K}=1$ if and only if exactly one $K_{i}$ is imaginary.

\section{Determination}

In this section, we determine all the imaginary number fields with class number one. Let $f$ be the conductor of $K$ and $t$ the number of prime divisors of $f$. We shall take generators $\chi_{i}$ of $X=\operatorname{Gal}(K / \mathbf{Q})^{\wedge}$ so that the number $r$ of them is minimal. For each prime divisor $p$ of $f$, let $X_{p}$ be the subgroup of $X$ consisting of characters of $p$-power conductors. For any odd $p, X_{p}$ is cyclic. Then, we take a generator of $X_{p}$ as $\chi_{i}$. On the other hand, $X_{2}$ is cyclic or a direct product of its two cyclic subgroups. When $f$ is even, we take also generator(s) of $X_{2}$ as $\chi_{i}$; if $X_{2}$ is not cyclic, i.e., if $K$ contains $\mathbf{Q}\left(\zeta_{2^{m}}\right)=\mathbf{Q}(\sqrt{-1}) \mathbf{Q}\left(\cos \left(\pi / 2^{m-1}\right)\right)$ for some $m \geqq 3$, then for simplicity, we take the character associated with $\mathbf{Q}(\sqrt{-1})$ as $\chi_{1}$, and a generator of the character group associated with $\mathbf{Q}\left(\cos \left(\pi / 2^{m-1}\right)\right)$ as $\chi_{2}$. (Then by Proposition U2, $Q_{K}=1$ if and only if exactly one of $\chi_{i}$ is odd.) Thus, $r=t+1$ or $t$, according as $K$ contains $\mathbf{Q}\left(\zeta_{2^{m}}\right)$ for some $m \geqq 3$ or not.

Now, we explain under which rule we examine all possible cases. First, we note that the character group associated with any subfield of $K$ with genus number one is of the form

$$
\left\langle\chi_{1}^{e_{1}}\right\rangle \times \cdots \times\left\langle\chi_{r}^{e_{r}}\right\rangle
$$

As described in the Introduction, we shall always take $\chi_{1}$ to be an odd generator

\footnotetext{
${ }^{5}$ Recently, Louboutin obtained sharper lower bounds for $h^{-}(K)([13,14])$, and if we had used them, we could have greatly reduced the amount of calculation in $\S 3$.
} 
of minimal order, and by $(*)$, in most cases, we can immediately restrict $K$ to be considered by using data on fields with smaller character group. Indeed, when $r \geqq 3$, the subfields of $K$ corresponding to

$$
\left\langle\chi_{1}\right\rangle \times \cdots \times\left\langle\chi_{r-1}\right\rangle \text { and }\left\langle\chi_{1}\right\rangle \times \cdots \times\left\langle\chi_{r-2}\right\rangle \times\left\langle\chi_{r}\right\rangle
$$

are imaginary abelian number fields with class number one by (\#). Hence, the values of $f_{\chi_{i}}$ are restricted by the data for $r-1$. In particular, if for some $r$ any $K$ with genus number one has class number greater than one, then so does any such $K$ for $r+1$. Therefore, it is reasonable to examine all possible cases according to the growth of $r$. Since $r=t+1$ or $t$, we shall examine them according to the growth of $t$, and for each $t$ first treat the case $r=t$ and then the case $r=t+1$. From the upper bound for conductors given by Uchida, we can get an upper bound for $t$, which is too large to examine all possible cases:

$$
\begin{aligned}
f<2 \times 10^{10} & \Longrightarrow 2^{2} \times \prod_{i=2}^{t} p_{i}<2 \times 10^{10}\left(p_{i}: \text { the } i \text { th prime }\right) \\
& \Longrightarrow t \leqq 10(\Longrightarrow r \leqq 11) .
\end{aligned}
$$

However, since the possibilities decrease fast according to the growth of $r$, it is expected that $r$ does not become so large. For each pair $(t, r)$, we first treat the cases where each ord $\chi_{i}$ is a prime (power) and then the other cases. Thus, the two basic cases are:

(i) the case $t=1$,

and

(ii) the case $t=r=2$, and ord $\chi_{1}$ is a power of two and ord $\chi_{2}$ is a power of two or an odd prime.

(Note that since we take $\chi_{1}$ odd, 2 always divides ord $\chi_{1}$.) Each case is divided into many subcases. Therefore, we must be careful. We divide each case into subcases according to the type of some subfield. We take as this subfield the maximal subfield of power-of-two degree $L$ in the two basic cases, and the subfield $F$ corresponding to $\left\langle\chi_{1}^{a}, \chi_{2}^{b}\right\rangle$ for some $a, b$ in the other cases.

Now, we determine the fields.

(i) We first treat the case $t=1$, i.e., $f=p^{m}$ ( $p$ : a prime). Since the cases where $n=[K: \mathbf{Q}]$ is a power of two have been treated by Uchida, it suffices to consider the cases where $n$ is not a power of two and $p$ is odd. Then $r=1$ and $K$ is cyclic. As we described in the Introduction, the possibilities of the values of $p$ are known by Uchida's result. In this case, for each $p$, we determine the fields as follows. Since any imaginary cyclic field of conductor $p^{m+1}$ has an imaginary subfield of conductor $p^{m}$, if $h(K)>1$ for all imaginary cyclic fields $K$ of conductor $p^{m}$, then also $h(K)>1$ for all such fields $K$ of conductor $p^{m+1}$ by $(*)$. Therefore, we first check whether or not $h(K)=1$ for all fields $K$ of conductor $p$. If there exists a field with $h(K)=1$ of conductor $p^{m}$, then we check whether or not $h^{-}(K)=1$ for all the fields $K$ of conductor $p^{m+1}$ containing that field. Suppose $2^{k} \| n$. Then $[L: \mathbf{Q}]=2^{k}$, and therefore $k=1,2,3$, or 4 by [30] (see Table 1). 
Assume $k=1$. Then $L$ is one of the imaginary quadratic number fields

$$
\mathbf{Q}(\sqrt{-d}): d=3,7,11,19,43,67,163 .
$$

Therefore, $p$ is one of the above $d$.

Assume $p=3$. All imaginary cyclic fields of power-of-three conductors are cyclotomic. Therefore, the determination has already been done in [21]. There exist exactly three imaginary cyclic number fields of power-of-three conductors with class number one, that is, $\mathbf{Q}(\sqrt{-3}), \mathbf{Q}\left(\zeta_{9}\right)$, and $\mathbf{Q}\left(\zeta_{27}\right)$.

Assume $p=7$. We know $h\left(\mathbf{Q}\left(\zeta_{7}\right)\right)=1$ and $h\left(\mathbf{Q}\left(\zeta_{49}\right)\right)>1$ [21]. We also know $h(K)=1$ for $f=7^{2}=49$ and $n=2 \cdot 7=14$ ([6, 20]). By the relative class number formula, we have $h^{-1}(K)>1$ for $f=7^{3}=343$ and $n=14 \cdot 7=98$. Thus, there exist exactly three imaginary cyclic fields of powerof-seven conductors and class number one, that is, $\mathbf{Q}(\sqrt{-7}), \mathbf{Q}\left(\zeta_{7}\right)$, and the subfield of $\mathbf{Q}\left(\zeta_{49}\right)$ of degree 14 .

The other values of $p$ are treated similarly.

For the other values of $k$, we first get a list of possible values of $p$ from the list of the imaginary cyclic number fields with class number one of degree $2^{k}$, and then treat each value of $p$ similarly.

Thus, together with Uchida's result, we get the following table.

TABLE 1

\begin{tabular}{|c|c|c|c|c|}
\hline$t$ & $r$ & Type & Degree & Fields \\
\hline \multirow{13}{*}{1} & & $2^{*}$ & 2 & $3 ; 4 ; 7 ; 8 ; 11 ; 19 ; 43 ; 67 ; 163$ \\
\hline & & $4^{*}$ & 4 & $5 ; 13 ; 16 ; 29 ; 37 ; 53 ; 61$ \\
\hline & & $6^{*}$ & 6 & $7 ; 9 ; 19 ; 43 ; 67$ \\
\hline & & $8^{*}$ & 8 & $32 ; 41$ \\
\hline & & $10^{*}$ & 10 & 11 \\
\hline & 1 & $12^{*}$ & 12 & $13 ; 37 ; 61$ \\
\hline & & $14^{*}$ & 14 & $43 ; 49$ \\
\hline & & $16^{*}$ & 16 & 17 \\
\hline & & $18^{*}$ & 18 & $19 ; 27$ \\
\hline & & $20^{*}$ & 20 & 25 \\
\hline & \multirow{3}{*}{2} & $\left(2^{*}, 2\right)$ & 4 & $(4,8)$ \\
\hline & & $\left(2^{*}, 4\right)$ & 8 & $(4,16)$ \\
\hline & & $\left(2^{*}, 8\right)$ & 16 & $(4,32)$ \\
\hline
\end{tabular}

In the table, ${ }^{*}$ means the oddness (imaginarity) of a generator of the character group $X_{p}$. Each field is expressed as $\left(f_{\chi_{1}}, \ldots, f_{\chi_{r}}\right)$ for each type $\left(\operatorname{ord} \chi_{1}, \ldots\right.$, ord $\left.\chi_{r}\right)$ of $X$. For example, for the field expressed as $(4,32)$ of type $\left(2^{*}, 8\right)$, the character group is generated by the odd character of order 2 and conductor 4 (say $\chi_{4}$ ), and an even character of order 8 and conductor 32 (say $\psi_{32}$ ). Hence, this field is the cyclotomic number field $\mathbf{Q}(\sqrt{-1}, \cos (2 \pi / 32))=\mathbf{Q}\left(\zeta_{32}\right)$. We note that this field is considered as also of type $\left(2^{*}, 8^{*}\right)$ by taking $\chi_{4}$ and $\chi_{4} \psi_{32}$ as generators of the character group. This is so in Uchida's table in [30].

(ii) Next we treat the case $t=r=2$ and ord $\chi_{1}=$ a power of two, ord $\chi_{2}=l$ (a power of two or an odd prime). The cases where $l$ is a power of two have 
been treated by Uchida. Therefore, we may assume that $l$ is an odd prime. Then $K$ is cyclic. Since $[L: \mathbf{Q}]=\operatorname{ord} \chi_{1}$, from Uchida's result (see Table 1), ord $\chi_{1}=2,4,8$, or 16 . For each ord $\chi_{1}$, the possibilities of the values of $f_{\chi_{1}}$ are known. In this case, for each $f_{\chi_{1}}$, we determine the fields as follows. By the estimate for $h^{-}(K)$ obtained in the previous section (or a better estimate), we get a lower bound for $l$ such that $h^{-}(K)>1$ for all $f_{x_{2}}$. Then for each fixed $f_{\chi_{1}}$ and $l$, we get an upper bound for $f_{\chi_{2}}$ for $h^{-}(K)>1$ by the same estimate. Then we calculate $h^{-}(K)$ for every $f_{x_{2}}\left(=p\right.$ or $l^{2}$, where $p$ is a prime with $p \equiv 1(\bmod l)$ and $\left.p \nmid f_{\chi_{1}}\right)$ less than the above upper bound. Then we check whether or not $h^{+}(K)=1$ for $K$ with $h^{-}(K)=1$.

Suppose ord $\chi_{1}=4$. Then, since $L$ is an imaginary cyclic quartic number field with class number one, from Table 1 ,

$$
f_{\chi_{1}}=5,13,16,29,37,53 \text {, or } 61 \text {. }
$$

Assume $f_{\chi_{1}}=5$. Then we have $w_{K}=10, D_{K}=\left(5^{3}\right)^{l} \cdot\left(f_{\chi_{2}}^{l-1}\right)^{4}$, and $D_{K^{+}}=$ $5^{l} \cdot\left(f_{\chi_{2}}^{l-1}\right)^{2}$. We also note that for the character $\chi=(\cdot / 5)$ associated with $\mathbf{Q}(\sqrt{5})$,

$$
L(1, \chi)=\frac{2 \log \{(\sqrt{5}+1) / 2\}}{\sqrt{5}}=0.4304 \ldots
$$

Therefore, we have

$$
h^{-}(K) \geqq \frac{10 \cdot 5^{l} \cdot f_{\chi_{2}}^{l-1}}{(2 \pi)^{2 l} \times 9.3 \times 0.431 \times c}
$$

where

$$
c=\left(\frac{1}{2} \log f_{\chi_{2}}+\gamma-\frac{1}{2}\right)^{l-1}\left(\frac{1}{2} \log 5 f_{\chi_{2}}+\gamma-\frac{1}{2}\right)^{l-1}\left(3 l \log 5+4(l-1) \log f_{\chi_{2}}\right) .
$$

From this, we have $h^{-}(K)>1$ for

$$
\begin{gathered}
l=3, f_{\chi_{2}} \geqq 2400 ; l=5, f_{\chi_{2}} \geqq 500 ; l=7, f_{\chi_{2}} \geqq 300 ; \\
l=11, f_{\chi_{2}} \geqq 150 ; l=13, f_{\chi_{2}} \geqq 110 ; l=17, f_{\chi_{2}} \geqq 90 ; \\
l=19, f_{\chi_{2}} \geqq 90 ; l=23, f_{\chi_{2}} \geqq 80 ; l=29, f_{\chi_{2}} \geqq 70 ; \\
l>29, \quad \text { for all } f_{\chi_{2}} .
\end{gathered}
$$

For each $l \leqq 29$, we calculate $h^{-}(K)$ by a computer for all $f_{\chi_{2}}$ less than the above upper bound; we find that $h^{-}(K)=1$ only for

$$
l=3, f_{\chi_{2}}=7 ; \quad l=3, f_{\chi_{2}}=9 .
$$

For these fields, $h^{+}(K)=1$ by [20]. For the other $f_{\chi_{1}}$, we have $h^{-}(K)>1$ for all $f_{\chi_{2}}$ by computer calculation.

The other values of ord $\chi_{1}$ are treated similarly.

Thus, together with Uchida's result, we get the following table . 
TABLE 2

\begin{tabular}{|c|c|c|c|c|}
\hline$t$ & $r$ & Type & Degree & Fields \\
\hline \multirow{10}{*}{2} & \multirow{10}{*}{2} & $\left(2^{*}, 2\right)$ & 4 & $\begin{array}{l}(3,5) ;(3,8) ;(3,17) ;(3,41) ;(3,89) ;(4,5) ;(4,13) ;(4,37) \\
(7,5) ;(7,13) ;(7,61) ;(8,5) ;(8,29) ;(11,8) ;(11,17)\end{array}$ \\
\hline & & $\left(2^{*}, 2^{*}\right)$ & 4 & $\begin{array}{l}(3,4) ;(3,7) ;(3,8) ;(3,11) ;(3,19) ;(3,19) ;(3,43) ;(3,67) ; \\
(3,163) ;(4,7) ;(4,11) ;(4,19) ;(4,43) ;(4,67) ;(4,163) \\
(7,8) ;(7,11) ;(7,19) ;(7,43) ;(7,163) ;(8,11) ;(8,19) ; \\
(8,43) ;(8,67) ;(11,19) ;(11,67) ;(11,163) ;(19,67) ; \\
(19,163) ;(43,67) ;(43,163) ;(67,163)\end{array}$ \\
\hline & & $\left(2^{*}, 3\right)$ & 6 & $\begin{array}{l}(3,7) ;(3,13) ;(3,31) ;(3,43) ;(4,7) ;(4,9) ;(4,19) ;(7,9) ; \\
(7,13) ;(8,7) ;(8,13) ;(11,7)\end{array}$ \\
\hline & & $\left(2^{*}, 4\right)$ & 8 & $(3,16)$ \\
\hline & & $\left(2^{*}, 4^{*}\right)$ & 8 & $\begin{array}{l}(3,5) ;(3,16) ;(4,5) ;(4,13) ;(4,37) ;(7,5) ;(7,13) ;(8,5) \\
(8,29) ;(11,16)\end{array}$ \\
\hline & & $\left(2^{*}, 5\right)$ & 10 & $(3,11) ;(4,11)$ \\
\hline & & $\left(2^{*}, 8^{*}\right)$ & 16 & $(3,32)$ \\
\hline & & $\left(4^{*}, 2\right)$ & 8 & $(5,8) ;(5,13) ;(5,17) ;(13,5) ;(13,8) ;(16,5)$ \\
\hline & & $\left(4^{*}, 3\right)$ & 12 & $(5,7) ;(5,9)$ \\
\hline & & $\left(4^{*}, 4^{*}\right)$ & 16 & $(5,13) ;(5,16)$ \\
\hline
\end{tabular}

The other cases are easily treated. We no longer need to use the estimate for $h^{-}(K)$. By the arithmetic formula and Proposition U2, we easily see that in some cases, $h^{-}(K)$ is a product of relative class numbers of two subfields, and therefore we immediately get the list of fields with $h^{-}(K)=1$ from the data on fields with smaller character group. Moreover, in some cases, for all fields $K$ satisfying (\#), $h^{-}(K)$ has been calculated already by Hasse [6] or Rechtenstamm [25]. But in some cases, we need to calculate $h^{-}(K)$ for many fields.

(iii) Next, we treat the case where $t=r=2$ and $K \neq L$ and ord $\chi_{1}$ is not a power of two or ord $\chi_{2}$ is a composite. Suppose $2^{k} \| \operatorname{ord} \chi_{1}$. Let $l$ be any prime divisor of ord $\chi_{2}$. Let $F$ be the subfield of $K$ corresponding to $\left\langle\chi_{1}^{\text {ord } \chi_{1} / 2^{k}}, \chi_{2}^{\text {ord } \chi_{2} / l}\right\rangle$. Then $F$ is an imaginary field of type $\left(\left(2^{k}\right)^{*}, l\right)$ with genus number one, and therefore $h(F)=1$ by (\#). Hence, from Table $2, k=1$ or 2 , and $l=2,3$, or 5 if $k=1$ and $l=2$, or 3 if $k=2$.

(a) Assume $k=1$, i.e., $2 \|$ ord $\chi_{1}$. Then from Table 1 , ord $\chi_{1}=2,6,10,14$, or 18 .

( $\alpha$ ) Suppose ord $\chi_{1}=2$. Then, since ord $\chi_{2}$ is not a power of two, 5, 6, or 9 divides ord $\chi_{2}$.

Suppose $5 \mid$ ord $\chi_{2}$. Then by the data on fields of type $\left(2^{*}, 5\right)$ and fields of power-of-eleven conductors, only the following two fields of type $\left(2^{*}, 10^{*}\right)$ satisfy (\#):

$$
\left(f_{\chi_{1}}, f_{\chi_{2}}\right)=(3,11),(4,11) \text {. }
$$

These are the cyclotomic fields $\mathbf{Q}\left(\zeta_{33}\right)$ and $\mathbf{Q}\left(\zeta_{44}\right)$ which have class number one [21].

Suppose $9 \mid \operatorname{ord} \chi_{2}$. Let $F$ be the subfield of $K$ corresponding to $\left\langle\chi_{1}\right.$, $\left.\chi_{2}^{\text {ord } \chi_{2} / 9}\right\rangle$. Then $F$ is an imaginary field of type $\left(2^{*}, 9\right)$ with genus number one. By the data on fields of type $\left(2^{*}, 3\right), F$ must be one of the fields 
with $\left(f_{\chi_{1}}, f_{\chi_{2}}\right)=(4,19),(4,27),(7,27)$. For these fields, by [25], $h^{-}(K)=$ $19,19,163$, respectively. Hence, we always have $h(K)>1$.

Suppose $6 \mid \operatorname{ord} \chi_{2}$ and $5,9 \nmid \operatorname{ord} \chi_{2}$, i.e., ord $\chi_{2}=6 \cdot 2^{m}$ for some $m \geq 0$. First, we assume that $\chi_{2}$ is an even character of order 6 . Then by the data on fields of types $\left(2^{*}, 2\right)$ and $\left(2^{*}, 3\right)$, only the field with $\left(f_{\chi_{1}}, f_{\chi_{2}}\right)=(7,13)$ satisfies (\#). But for this field, $h^{-}(K)=7$ by [6]. Hence, when $m>0$, we always have $h(K)>1$. Thus, we may assume that $\chi_{2}$ is an odd character of order 6 . Let $F_{1}$ (resp. $F_{2}$ ) be the subfield of $K$ corresponding to $\left\langle\chi_{1}, \chi_{2}^{2}\right\rangle$ (resp. $\left.\left\langle\chi_{2}\right\rangle\right)$. Then it is easily seen that $h^{-}(K)=h^{-}\left(F_{1}\right) h^{-}\left(F_{2}\right)$. Therefore, by the data on sextic fields, $h^{-}(K)=1$ exactly for

$$
\left(f_{\chi_{1}}, f_{\chi_{2}}\right)=(3,7),(3,43),(4,7),(4,9),(4,19),(7,9),(8,7),(11,7) \text {. }
$$

For all these fields, $h^{+}(K)=1$ : The first, third, and fourth fields are the cyclotomic fields $\mathbf{Q}\left(\zeta_{21}\right), \mathbf{Q}\left(\zeta_{28}\right)$, and $\mathbf{Q}\left(\zeta_{36}\right)$, which have class number one [21]; for the second field this follows by [16], and for the others by [20].

Thus, if ord $\chi_{1}=2$ and ord $\chi_{2}$ is composite and not a power of two, $h(K)=$ 1 exactly for the above ten fields.

( $\beta$ ) Suppose ord $\chi_{1}=6$. Then we may assume $\chi_{2}$ is even or ord $\chi_{2}$ is not a power of two $>8$.

Suppose $\chi_{2}$ is an even character of order two. Therefore, by the data on fields of type $\left(2^{*}, 2\right)$, only the following eight fields satisfy $(\#)$ :

$$
\left(f_{\chi_{1}}, f_{\chi_{2}}\right)=(7,5),(7,13),(7,61),(9,5),(9,8),(9,17),(9,41),(9,89) \text {. }
$$

For these fields, by computer calculation,

$$
h^{-}(K)=1,4,9,1,1,4,19,28,
$$

respectively. For the above three fields with $h^{-}(K)=1$, we get $h^{+}(K)=1$ by [20]. From this and the data on fields of type $\left(2^{*}, 5\right)$, if there exists another field with $h(K)=1$, ord $\chi_{1}=6$, and $2 \mid$ ord $\chi_{2}$, then it contains the field with $\left(f_{\chi_{1}}, f_{\chi_{2}}\right)=(9,16)$ and ord $\chi_{2}=4$. But for this field, $h^{-}(K)=13$ by [25]. Therefore, such a field does not exist.

Suppose ord $\chi_{2}=3$. Then by the data on fields of type $\left(2^{*}, 3\right)$, only the following six fields satisfy (\#):

$$
\left(f_{\chi_{1}}, f_{\chi_{2}}\right)=(7,9),(7,13),(9,7),(9,13),(9,31),(9,43) \text {. }
$$

For these fields, by [25],

$$
h^{-}(K)=7,13,1,7,91,247,
$$

respectively. For the above field with $h^{-}(K)=1$, we have $h^{+}(K)=1$ by [11]. Since there exists no field of type $\left(2^{*}, 7\right)$ with $h^{-}(K)=1$, among the fields with ord $\chi_{1}=6$, and $3 \mid$ ord $\chi_{2}$, only this field has class number one.

Suppose ord $\chi_{2}=5$. Then by the data on fields of type $\left(2^{*}, 5\right)$, only the field with $\left(f_{\chi_{1}}, f_{\chi_{2}}\right)=(9,11)$ satisfies $(\#)$. But for this field, $h^{-}(K)=31$ by [6]. Thus, there exists no field with $5 \mid$ ord $\chi_{2}$ and $h(K)=1$.

$(\gamma)$ The cases ord $\chi_{1}=10,14$, and 18 are treated similarly, and in these cases we always have $h(K)>1$. 20.

(b) Assume $k=2$, i.e., $4 \| \operatorname{ord} \chi_{1}$. Then from Table 1, ord $\chi_{1}=4,12$, or

( $\alpha$ ) Suppose ord $\chi_{1}=4$. Then, from Table 2, $3 \mid \operatorname{ord} \chi_{2}$. 
Suppose $\operatorname{ord} \chi_{2}=6$. Then by the data on fields of type $\left(4^{*}, 3\right)$, only the following two fields satisfy (\#):

$$
\left(f_{\chi_{1}}, f_{\chi_{2}}\right)=(5,7),(5,9) \text {. }
$$

These two fields are the cyclotomic fields $\mathbf{Q}\left(\zeta_{35}\right)$ and $\mathbf{Q}\left(\zeta_{45}\right)$, which have class number one [20].

Suppose ord $\chi_{2}=9$. Then by the data on fields of type $\left(4^{*}, 3\right)$, only the field with $\left(f_{\chi_{1}}, f_{\chi_{2}}\right)=(5,27)$ satisfies (\#). But for this field, $h^{-}(K)=2053$ by [25]. Thus, since $h^{-}(K)>1$ for any field $K$ of type $\left(4^{*}, 7\right)$, if ord $\chi_{1}=4$, we have $h(K)=1$ only for the above two fields.

$(\beta)$ The cases ord $\chi_{1}=12$ and 20 are treated similarly, and in these cases we always have $h(K)>1$. Thus, we get the following table.

TABLE 3

\begin{tabular}{|l|l|l|l|l|}
\hline$t$ & $r$ & Type & Degree & \multicolumn{1}{c|}{ Fields } \\
\hline \hline \multirow{4}{*}{2} & & $\left(2^{*}, 6^{*}\right)$ & 12 & $(3,7) ;(3,43) ;(4,7) ;(4,9) ;(4,19) ;(7,9) ;(8,7) ;(11,7)$ \\
\cline { 3 - 5 } & \multirow{2}{*}{2} & $\left(2^{*}, 10^{*}\right)$ & 20 & $(3,11) ;(4,11)$ \\
\cline { 2 - 5 } & $\left(4^{*}, 6^{*}\right)$ & 24 & $(5,7) ;(5,9)$ \\
\cline { 3 - 5 } & & $\left(6^{*}, 2\right)$ & 12 & $(7,5) ;(9,5) ;(9,8)$ \\
\cline { 3 - 5 } & & $\left(6^{*}, 3\right)$ & 18 & $(9,7)$ \\
\hline
\end{tabular}

(iv) Next, we treat the case where $t=2$ and $r=3$. Let $F$ be the subfield corresponding to $X_{2}=\left\langle\chi_{1}, \chi_{2}\right\rangle$. Then, from Table $1, F=\mathbf{Q}\left(\zeta_{8}\right), \mathbf{Q}\left(\zeta_{16}\right)$, or $\mathbf{Q}\left(\zeta_{32}\right)$. We consider only the cases where $L \neq K$. We put ord $\chi_{3}=q$.

(a) Assume $F=\mathbf{Q}\left(\zeta_{8}\right)$. Then both of the subfields corresponding to $\left\langle\chi_{1}, \chi_{3}\right\rangle$, $\left\langle\chi_{1} \chi_{2}, \chi_{3}\right\rangle$ are imaginary fields of type $\left(2^{*}, q\right)$ with class number one, which are known in (ii) and (iii). Thus, we have $q=3$ or 6 from Tables 2 and 3. When $q=3$, from Table 3, only the field with $f_{\chi_{3}}=7$ satisfies (\#). For this field, we know $h^{-}(K)=1$ by [6] and $h^{+}(K)=1$ by [20]. When $q=6$, the subfields corresponding to $\left\langle\chi_{1}, \chi_{2}, \chi_{3}^{2}\right\rangle$ is the above field. Therefore, $f_{\chi_{3}}=7$. Hence, $K=\mathbf{Q}\left(\zeta_{56}\right)$, for which we know $h(K)>1$ by [21].

(b) Assume $F=\mathbf{Q}\left(\zeta_{16}\right)$. Then the subfield corresponding to $\left\langle\chi_{1}, \chi_{2}^{2}, \chi_{3}\right\rangle$ is the above field $\mathbf{Q}\left(\zeta_{8}, \cos (2 \pi / 7)\right)$. Therefore, $q=3$ and $f_{\chi_{3}}=7$. For this field, $h^{-}(K)=9$ by [25].

(c) Similarly, if $F=\mathbf{Q}\left(\zeta_{32}\right)$, we have $h(K)>1$ for any $\chi_{3}$.

Thus, together with Uchida's result, we get the following table.

TABLE 4

\begin{tabular}{|l|c|c|c|l|}
\hline$t$ & $r$ & Type & Degree & \\
\hline \hline \multirow{4}{*}{2} & \multirow{4}{*}{3} & $\left(2^{*}, 2,2\right)$ & 8 & $(4,8,5)$ \\
\cline { 3 - 5 } & & $\left(2^{*}, 2,2^{*}\right)$ & 8 & $(4,8,3) ;(4,8,11)$ \\
\cline { 3 - 5 } & $\left(2^{*}, 2,3\right)$ & 12 & $(4,8,7)$ \\
\cline { 3 - 5 } & $\left(2^{*}, 2,4^{*}\right)$ & 16 & $(4,8,5)$ \\
\cline { 3 - 5 } & $\left(2^{*}, 4,2\right)$ & 16 & $(4,16,5)$ \\
\cline { 3 - 5 } & & $\left(2^{*}, 4,2^{*}\right)$ & 16 & $(4,16,3)$ \\
\hline
\end{tabular}


(v) Next, we treat the case where $t=r=3$. Let $F$ be the subfield of $K$ corresponding to $\left\langle\chi_{1}, \chi_{2}\right\rangle$ and put ord $\chi_{3}=q$.

(a) Suppose that $F$ is of type $\left(2^{*}, 2\right)$. The cases where $q$ is a power of two have been treated by Uchida. In the other cases, from Tables 2 and $3, q=3,5$, or 6 .

$(\alpha)$ Assume $q=3$. Then by the data on fields of types $\left(2^{*}, 2\right)$ and $\left(2^{*}, 3\right)$, (\#) is satisfied only by the fields with

$$
\begin{aligned}
\left(f_{\chi_{1}}, f_{\chi_{2}}, f_{\chi_{3}}\right)= & (3,5,7),(3,5,13),(3,5,31),(3,5,43),(3,8,7), \\
& (3,8,13),(3,8,31),(3,8,43),(3,17,7),(3,17,13), \\
& (3,17,31),(3,17,43),(3,41,7),(3,41,13),(3,41,31), \\
& (3,41,43),(3,89,7),(3,89,13),(3,89,31),(3,89,43), \\
& (4,5,7),(4,5,9),(4,5,19),(4,13,7),(4,13,9), \\
& (4,13,19),(4,37,7),(4,37,9),(4,37,19),(7,5,9), \\
& (7,5,13),(7,13,9),(7,61,9),(7,61,13),(8,5,7), \\
& (8,5,13),(8,29,7),(8,29,13),(11,8,7),(11,17,7) .
\end{aligned}
$$

For these fields, by computer calculation,

$$
\begin{aligned}
h^{-}(K)= & 1,4,63,61,3,13,12,28,13,27,13,27,13,27,28,37,63, \\
& 84,91,91,196,175,3,7,7,12,13,63,31,52,84,9,12,4, \\
& 211,181,9,12,52,9,57,57,
\end{aligned}
$$

respectively. For the first field, $h^{+}(K)=1$ by [20].

$(\beta)$ Assume $q=5$. Then by the data on fields of types $\left(2^{*}, 2\right)$ and $\left(2^{*}, 5\right)$, only the following eight fields satisfy (\#):

$$
\begin{aligned}
\left(f_{\chi_{1}}, f_{\chi_{2}}, f_{\chi_{3}}\right)= & (3,5,11),(3,8,11),(3,17,11),(3,41,11),(3,89,11), \\
& (4,5,11),(4,13,11),(4,37,11) .
\end{aligned}
$$

For these fields, by computer calculation,

$$
h^{-}(K)=11,25,355,1705,15856,31,155,3971,
$$

respectively.

( $\gamma$ ) Assume $q=6$. Then the subfield of $K$ corresponding to $\left\langle\chi_{1}, \chi_{2}, \chi_{3}^{2}\right\rangle$ must be the field obtained when $q=3$. Therefore, $\left(f_{\chi_{1}}, f_{\chi_{2}}, f_{\chi_{3}}\right)=(3,5,7)$. For this field, $h^{-}(K)=1$ by [25] and $h^{+}(K)=1$ by [11].

(b) Suppose that $F$ is of type $\left(2^{*}, 2^{*}\right)$. The cases where $q$ is a power of two have been treated by Uchida. In the other cases, from Tables 2 and 3, and the data on fields of type $\left(2^{*}, 2^{*}, 2^{*}\right)$ in [28] (see Table 5 below), $q=3,5,6$, or 10 . Let $E$ be the subfield of $K$ corresponding to $\left\langle\chi_{1}, \chi_{3}\right\rangle$. Then, when $\chi_{3}$ is even, it is easily seen that $h^{-}(K)=h^{-}(F) h^{-}(E)$. Therefore, we can get the fields with $h^{-}(K)=1$ immediately from Table 2 .

( $\alpha$ ) Assume $q=3$. Then $h^{-}(K)=1$ exactly for

$$
\begin{aligned}
\left(f_{\chi_{1}}, f_{\chi_{2}}, f_{\chi_{3}}\right)= & (3,4,7),(3,7,13),(3,8,7),(3,8,13),(3,11,7), \\
& (4,7,9),(4,11,7),(7,8,13),(8,11,7) .
\end{aligned}
$$

For all these fields except the last field for which $h^{+}(K)=3$, we have $h^{+}(K)=1$ by $[16]$. 
( $\beta$ ) Assume $q=5$. Then $h^{-}(K)=1$ only for $\left(f_{\chi_{1}}, f_{\chi_{2}}, f_{\chi_{3}}\right)=(3,4,11)$. For this field, $h^{+}(K)=1$ by [11].

( $\gamma$ ) Assume $q=6$. Then $\chi_{3}$ is odd. By the data on fields of types $\left(2^{*}, 6^{*}\right)$ and $\left(2^{*}, 2^{*}, 2^{*}\right)$, only the following three fields satisfy (\#):

$$
\left(f_{\chi_{1}}, f_{\chi_{2}}, f_{\chi_{3}}\right)=(3,4,7),(3,8,7),(4,7,9) .
$$

The first field is the cyclotomic field $\mathbf{Q}\left(\zeta_{84}\right)$, which has class number one [21]. For the second and third fields, by [25] $h^{-}(K)=7,71344$, respectively.

$(\delta)$ Assume $q=10$. Then the subfield of $K$ corresponding to $\left\langle\chi_{1}, \chi_{2}, \chi_{3}^{2}\right\rangle$ must be the field obtained when $q=5$. Therefore, $\left(f_{\chi_{1}}, f_{\chi_{2}}, f_{\chi_{3}}\right)=(3,4,11)$. But this field is the cyclotomic field $\mathbf{Q}\left(\zeta_{132}\right)$ which has class number greater than one [21].

(c) Suppose that $F$ is of type $\left(2^{*}, 3\right)$ and $q \geqq 3$. Then from Tables 2 and $3, q=3,4,5$, or 6 .

( $\alpha$ ) Assume $q=3$. Then by the data on fields of type $\left(2^{*}, 3\right)$, only the following eleven fields satisfy (\#):

$$
\begin{aligned}
\left(f_{\chi_{1}}, f_{\chi_{2}}, f_{\chi_{3}}\right)= & (3,7,13),(3,7,31),(3,7,43),(3,13,31),(3,13,43), \\
& (3,31,43),(4,7,9),(4,7,19),(4,9,19),(7,9,13), \\
& (8,7,13) .
\end{aligned}
$$

For these fields, by computer calculation,

$$
h^{-}(K)=36,324,1008,2736,5292,3024,28,196,592,2128,1072,
$$

respectively.

( $\beta$ ) Assume $q=4$. Let $F_{1}$ (resp. $F_{2}$ ) be the subfield of $K$ corresponding to $\left\langle\chi_{1}, \chi_{3}^{2}, \chi_{2}\right\rangle$ (resp. $\left.\left\langle\chi_{3}, \chi_{2}\right\rangle\right)$. Then it is easily seen that $h^{-}(K)=$ $h^{-}\left(F_{1}\right) h^{-}\left(F_{2}\right)$. Therefore, by the data on fields of types $\left(2^{*}, 2,3\right)$ and $\left(4^{*}, 3\right)$, $h^{-}(K)=1$ only for

$$
\left(f_{\chi_{1}}, f_{\chi_{2}}, f_{\chi_{3}}\right)=(3,7,5) .
$$

For this field, $h^{+}(K)=1$ by [11].

$(\gamma)$ Assume $q=5$. Then by the data on fields of types $\left(2^{*}, 3\right)$ and $\left(2^{*}, 5\right)$, only the following seven fields satisfy (\#):

$$
\begin{aligned}
\left(f_{\chi_{1}}, f_{\chi_{2}}, f_{\chi_{3}}\right)= & (3,7,11),(3,13,11),(3,31,11),(3,43,11),(4,7,11), \\
& (4,9,11),(4,19,11) .
\end{aligned}
$$

For these fields, by computer calculation,

$$
h^{-}(K)=976,17701,515401,4733721,2896,19231,449296 \text {, }
$$

respectively.

$(\delta)$ Assume $q=6$. Then by the data on fields of type $\left(2^{*}, 3,3\right)$, we always have $h(K)>1$.

(d) The other types of $F$ are treated similarly, and we do not get another field with class number one.

Thus, together with Uchida's result, we get the following table . 
TABLE 5

\begin{tabular}{|c|c|c|c|l|}
\hline$t$ & $r$ & Type & Degree & \multicolumn{1}{c|}{ Fields } \\
\hline \hline \multirow{5}{*}{3} & \multirow{4}{*}{3} & $\left(2^{*}, 2,2^{*}\right)$ & 8 & $\begin{array}{l}(3,5,4) ;(3,5,7) ;(3,5,8) ;(3,8,11) ;(3,17,11) ;(4,5,7) ; \\
(4,13,7) ;(7,5,8)\end{array}$ \\
\cline { 3 - 5 } & \multirow{5}{*}{3} & $\left(2^{*}, 2,3\right)$ & 12 & $(3,5,7)$ \\
\cline { 3 - 5 } & $\left(2^{*}, 2,6^{*}\right)$ & 24 & $(3,5,7)$ \\
\cline { 3 - 5 } & $\left(2^{*}, 2^{*}, 2^{*}\right)$ & 8 & $(3,4,7) ;(3,4,11) ;(3,4,19) ;(3,7,8) ;(3,11,19) ;(4,7,19)$ \\
\cline { 3 - 5 } & $\left(2^{*}, 2^{*}, 3\right)$ & 12 & $\begin{array}{l}(3,4,7) ;(3,7,13) ;(3,8,7) ;(3,8,13) ;(3,11,7) ;(4,7,9) ; \\
(4,11,7) ;(7,8,13)\end{array}$ \\
\cline { 3 - 5 } & $\left(2^{*}, 2^{*}, 4^{*}\right)$ & 16 & $(3,4,5) ;(3,7,5) ;(3,8,5) ;(4,7,5)$ \\
\cline { 3 - 5 } & $\left(2^{*}, 2^{*}, 5\right)$ & 20 & $(3,4,11)$ \\
\cline { 3 - 5 } & $\left(2^{*}, 2^{*}, 6^{*}\right)$ & 24 & $(3,4,7)$ \\
\cline { 3 - 5 } & $\left(2^{*}, 3,4^{*}\right)$ & 24 & $(3,7,5)$ \\
\hline
\end{tabular}

(vi) Next, we treat the case where $t=3$ and $r=4$. In this case, from Tables 4 and 5 , we always have $h(K)>1$. Therefore, if $r=t+1 \geqq 4$, then always $h(K)>1$.

(vii) Next, we treat the case where $t=r=4$. We note that by [29], the 2rank of $X$ is at most three. Therefore, from Table 5, we conclude that $K$ is of type $\left(2^{*}, 2^{*}, 2^{*}, 3\right)$ and $\left(f_{\chi_{1}}, f_{\chi_{2}}, f_{\chi_{3}}, f_{\chi_{4}}\right)=(3,4,11,7)$, or $(3,7,8,13)$. For the first field, $h^{-}(K)=27$ by [25]. For the second field, $h^{-}(F)=39$ by computer calculation, whence $39 \mid h^{-}(K)$, where $F$ is the sextic subfield of $K$ corresponding to $\left\langle\chi_{1} \chi_{2} \chi_{3}, \chi_{4}\right\rangle$.

Thus, if $r \geqq 4$, then we always have $h(K)>1$.

The determination is now complete.

\section{APPENDIX. ON UNRAMIFIED-CLOSED ALGEBRAIC NUMBER FIELDS}

In this appendix, we denote by $K$ an algebraic number field of finite degree. Put $n_{K}=[K: \mathbf{Q}]$ and let $r_{1}(K)$ (resp. $r_{2}(K)$ ) be the number of real (resp. imaginary) primes of $K$. We call $K$ unramified-closed if $K$ does not have any nontrivial unramified extension. Here, the unramifiedness refers also to the infinite primes. It is well known that $\mathbf{Q}$ is unramified-closed as a corollary to Minkowski's theorem. We know some other unramified-closed fields (see [10, 23, 31, Exercise 11.2]). As we review below, if the root discriminant $r d_{K}$ of $K$ is sufficiently small, then $K$ is unramified-closed. Here,

$$
r d_{K}=D_{K}^{1 / n_{K}},
$$

where $D_{K}$ is the absolute value of the discriminant of $K$. Many of imaginary abelian number fields with class number one are unramified-closed:

(**) Among the 172 fields, at least 132 fields are unramified-closed, and if the Generalized Riemann Hypothesis (GRH) is true, then at least 155 fields are unramified-closed.

Now we review the basic idea to get unramified-closed fields. We first note that if $K$ is unramified-closed, then the class number $h(K)$ is one. We use the following four lemmas and lower estimates for root discriminants due to Odlyzko [24]. 
Lemma 1. Any finite group of order less than 60 is solvable. $\left(A_{5}\right.$, the alternating group of degree five whose order is 60 , is nonsolvable.)

Lemma 2. Let $L / K$ be an extension unramified at all finite primes. Then $r d_{L}=$ $r d_{K}$. (See, for example, [19].)

Lemma 3. Let $B\left(n, r_{1}, r_{2}\right)$ be the lower bound for root discriminants of algebraic number fields $L$ of degree $(\geqq n)$ such that $r_{i}(L) / n_{L}=r_{i} / n \quad(i=1,2)$. If $h(K)=1$ and $r d_{K}<B\left(60 n_{K}, 60 r_{1}(K), 60 r_{2}(K)\right)$, then $K$ is unramified-closed.

Proof. Suppose that $K$ has a nontrivial unramified extension $M$. Then the Galois closure $L$ of $M$ over $K$ is also unramified over $K$. By Lemma $2, r d_{L}=$ $r d_{K}$. Since $r_{i}(L)=[L: K] r_{i}(K) \quad(i=1,2)$, we have $r_{i}(L) / n_{L}=r_{i}(K) / n_{K}$. If $\mathrm{Gal}(L / K)$ is solvable, then $K$ has a nontrivial unramified abelian extension, which is contrary to $h(K)=1$. Therefore, $\operatorname{Gal}(L / K)$ is nonsolvable. Hence, by Lemma 1 , we have $[L: K]=\# \operatorname{Gal}(L / K) \geqq 60$. Thus, this contradicts the assumption. Hence, $K$ is unramified-closed.

Remark. We also know that if $r d_{K}<B\left(h n_{K}, h r_{1}(K), h r_{2}(K)\right)$, then $h(K)<$ $h$. (See, for example, [31, Lemma 11.23].) Thus, in particular, if $r d_{K}<$ $B\left(2 n_{K}, 2 r_{1}(K), 2 r_{2}(K)\right)$, then $K$ has class number one. This idea is very useful for calculation of class numbers. Indeed, it is used together with some algebraic technique for calculation of real abelian number fields ([11, 20]).

Odlyzko gave good lower bounds for $B\left(n, r_{1}, r_{2}\right)$ (unconditional and conditional (under GRH) ones) [24]. (See [1], or [18].) We now give a table of lower bounds for $B(60 n, 0,30 n)$ which is copied from Diaz y Diaz [1] (unconditional) and Odlyzko [24] (conditional).

Lower bounds for $B(60 n, 0,30 n)$

\begin{tabular}{|r|c|c|c|}
\hline \multicolumn{1}{|c|}{$n$} & $60 n$ & unconditional & under GRH \\
\hline 2 & 120 & 17.05391605 & 20.221 \\
4 & 240 & 18.81483169 & 23.575 \\
6 & 360 & 19.59036104 & 25.332 \\
8 & 480 & 20.04432277 & 26.485 \\
10 & 600 & 20.34836535 & 27.328 \\
12 & 720 & 20.56889558 & 27.984 \\
14 & 840 & 20.73755115 & 28.515 \\
16 & 960 & 20.87150629 & 28.961 \\
18 & 1080 & 20.98096539 & \\
20 & 1200 & 21.07240885 & 29.673 \\
24 & 1440 & 21.21725941 & \\
\hline
\end{tabular}

(Conditional lower bounds for $B(1080,0,540)$ and $B(1440,0,720)$ are not given in [24]. But we do not need these values for the confirmation of $(* *)$.)

By Lemma 3, (**) is confirmed by calculating root discriminants. ${ }^{6}$ For their calculation, it is convenient to use the following:

\footnotetext{
${ }^{6}$ We give approximate values of root discriminants of the imaginary abelian number fields with class number one below.
} 
Lemma 4. Let $E$ and $F$ be algebraic number fields with relatively prime discriminants, and $K$ the composite field of $E$ and $F$. Then $r d_{K}=r d_{E} r d_{F}$. (See, for example, [20].)

Since any imaginary abelian number field with genus number one is a compositum of fields of prime power conductors which are pairwise relatively prime, it is easy to calculate its root discriminant.

We propose the following question:

"Is any imaginary abelian number field with class number one unramified-closed?"

Unfortunately, we cannot answer this question only by estimating root discriminants, because we do not have any means of judging whether or not $K$ is unramified-closed when $h(K)=1$ and $r d_{K} \geqq B\left(60 n_{K}, 60 r_{1}(K), 60 r_{2}(K)\right)$. Note also that we have the following examples:

Example 1. The imaginary biquadratic bicyclic number field $\mathbf{Q}(\sqrt{-67}, \sqrt{-163})$ has class number one. We do not know whether or not this field is unramifiedclosed. Its root discriminant is $\sqrt{67 \cdot 163}=104.5 \ldots$.

Example 2 (Martinet [17]). The imaginary cyclic number field $\mathbf{Q}\left(\cos \frac{2}{11} \pi, \sqrt{-46}\right)$ has an infinite class field tower. The root discriminant of this field is $2^{3 / 2} \cdot 23^{1 / 2}$. $11^{4 / 5}=92.368 \ldots$

There exist many real quadratic number fields with class number one having nontrivial unramified extension (see $[32,33]$ ). But still unknown is any example of such an imaginary abelian number field. We also note that many imaginary abelian number fields with small class number do not have an unramified nonsolvable Galois extension. We have the following example:

Example 3. The imaginary quadratic number field $\mathbf{Q}(\sqrt{-4903})$ has an unramified extension whose Galois group is isomorphic to $A_{5}$. Such an extension is given as the splitting field of the polynomial $X^{5}+X^{4}-3 X^{3}-X^{2}+2 X-1$. The class number of $\mathbf{Q}(\sqrt{-4903})$ is 27 . Its root discriminant is $70.02 \ldots$ (For unramified $A_{n}$-extensions ${ }^{7}$ of quadratic number fields, see [32] or [34].)

We now give the table of imaginary abelian number fields with class number one, in which fields are ordered by their degrees and conductors. We use the following notations in order to express generators of associated character groups. $\chi_{4}$ denotes the unique primitive Dirichlet character of conductor 4 . For odd prime numbers $p, \chi_{p}$ denotes a primitive Dirichlet character of conductor $p$ and order $p-1$. For prime powers $q=p^{m} \quad(\neq 4), \psi_{q}$ denotes an even primitive Dirichlet character of conductor $q$ and order $p^{m-1}$ or $2^{m-2}$ according as $p$ is odd or $p=2$. In the column UC, the letter $\mathrm{Y}$ (resp. (Y)) means unramified-closed (resp. unramified-closed under GRH).

\footnotetext{
${ }^{7}$ Galois extensions whose Galois groups are isomorphic to $A_{n}$, the alternating group of degree $n$.
} 
Table of the imaginary abelian number fields with class number one

\begin{tabular}{|c|c|c|c|c|c|c|}
\hline$n$ & Type & Generators & $f$ & $r d$ & Simple expression & UC \\
\hline & \multirow{9}{*}{$2^{*}$} & $\chi_{3}$ & 3 & 1.73 & $\mathbf{Q}(\sqrt{-3})$ & $Y$ \\
\hline & & $\chi_{4}$ & 4 & 2.00 & $\mathbf{Q}(\sqrt{-1})$ & $\mathrm{Y}$ \\
\hline & & $\chi_{7}^{3}$ & 7 & 2.64 & $\mathbf{Q}(\sqrt{-7})$ & $\bar{Y}$ \\
\hline & & $\chi_{4} \psi_{8}$ & 8 & 2.82 & $\mathbf{Q}(\sqrt{-2})$ & $\mathrm{Y}$ \\
\hline 2 & & $\chi_{11}^{5}$ & 11 & 3.31 & $\mathbf{Q}(\sqrt{-11})$ & $\mathrm{Y}$ \\
\hline & & $\chi_{19}^{9}$ & 19 & 4.35 & $\mathbf{Q}(\sqrt{-19})$ & Y \\
\hline & & $\chi_{43}^{21}$ & 43 & 6.55 & $Q(\sqrt{-43})$ & $\mathrm{Y}$ \\
\hline & & $\chi_{67}^{33}$ & 67 & 8.18 & $\mathbf{Q}(\sqrt{-67})$ & $\bar{Y}$ \\
\hline & & $\chi_{163}^{81}$ & 163 & 12.76 & $\mathbf{Q}(\sqrt{-163})$ & $\mathbf{Y}$ \\
\hline \multirow{23}{*}{4} & \multirow{7}{*}{$4^{*}$} & $\chi_{5}$ & 5 & 3.34 & $\mathbf{Q}\left(\zeta_{5}\right)$ & $\mathrm{Y}$ \\
\hline & & $\chi_{13}^{3}$ & 13 & 6.84 & $\mathbf{Q}(\sqrt{-(13+2 \sqrt{13})})$ & $\mathbf{Y}$ \\
\hline & & $\chi_{4} \psi_{16}$ & 16 & 6.72 & $\mathbf{Q}(i \sin (\pi / 8))$ & $\mathrm{Y}$ \\
\hline & & $\chi_{29}^{7}$ & 29 & 12.49 & $\mathbf{Q}(\sqrt{-(29+2 \sqrt{29})})$ & $\mathbf{Y}$ \\
\hline & & $\chi_{37}^{9}$ & 37 & 15.00 & $\mathbf{Q}(\sqrt{-(37+6 \sqrt{37})})$ & $\mathbf{Y}$ \\
\hline & & $\chi_{53}^{13}$ & 53 & 19.64 & $Q(\sqrt{-(53+2 \sqrt{53}}))$ & $(\mathrm{Y})$ \\
\hline & & $\chi_{61}^{15}$ & 61 & 21.82 & $\mathbf{Q}(\sqrt{-(61+6 \sqrt{61})})$ & $(\mathrm{Y})$ \\
\hline & \multirow{16}{*}{$\left(2^{*}, 2\right)$} & $\chi_{4}, \psi_{8}$ & 8 & 4.00 & $\mathbf{Q}\left(\zeta_{8}\right)$ & $\mathrm{Y}$ \\
\hline & & $\chi_{3}, \chi_{5}^{2}$ & 15 & 3.87 & $\mathbf{Q}(\sqrt{-3}, \sqrt{5})$ & $\mathrm{Y}$ \\
\hline & & $\chi_{4}, \chi_{5}^{2}$ & 20 & 4.47 & $\mathbf{Q}(\sqrt{-1}, \sqrt{5})$ & Y \\
\hline & & $\chi_{3}, \psi_{8}$ & 24 & 4.89 & $\mathbf{Q}(\sqrt{-3}, \sqrt{2})$ & $Y$ \\
\hline & & $\chi_{7}^{3}, \chi_{5}^{2}$ & 35 & 5.91 & $\mathbf{Q}(\sqrt{-7}, \sqrt{5})$ & $Y$ \\
\hline & & $\chi_{4} \psi_{8}, \chi_{5}^{2}$ & 40 & 6.32 & $\mathbf{Q}(\sqrt{-2}, \sqrt{5})$ & $\mathrm{Y}$ \\
\hline & & $\chi_{3}, \chi_{17}^{8}$ & 51 & 7.14 & $\mathbf{Q}(\sqrt{-3}, \sqrt{17})$ & $\mathbf{Y}$ \\
\hline & & $\chi_{4}, \chi_{13}^{6}$ & 52 & 7.21 & $\mathbf{Q}(\sqrt{-1}, \sqrt{13})$ & $\mathrm{Y}$ \\
\hline & & $\chi_{11}^{5}, \psi_{8}$ & 88 & 9.38 & $\mathbf{Q}(\sqrt{ }-11, \sqrt{2})$ & $\mathrm{Y}$ \\
\hline & & $\chi_{7}^{3}, \chi_{13}^{6}$ & 91 & 9.53 & $\mathbf{Q}(\sqrt{-7}, \sqrt{13})$ & $\mathbf{Y}$ \\
\hline & & $\chi_{3}, \chi_{41}^{20}$ & 123 & 11.09 & $\mathbf{Q}(\sqrt{-3}, \sqrt{41})$ & $Y$ \\
\hline & & $\chi_{4}, \chi_{37}^{18}$ & 148 & 12.16 & $\mathbf{Q}(\sqrt{-1}, \sqrt{37})$ & $Y$ \\
\hline & & $\chi_{11}^{5}, \chi_{17}^{8}$ & 187 & 13.67 & $\mathbf{Q}(\sqrt{-11}, \sqrt{17})$ & $Y$ \\
\hline & & $\chi_{4} \psi_{8}, \chi_{29}^{14}$ & 232 & 15.23 & $\mathbf{Q}(\sqrt{-2}, \sqrt{29})$ & $\mathrm{Y}$ \\
\hline & & $\chi_{3}, \chi_{89}^{44}$ & 267 & 16.34 & $\mathbf{Q}(\sqrt{-3}, \sqrt{89})$ & $\mathrm{Y}$ \\
\hline & & $\chi_{7}^{3}, \chi_{61}^{33}$ & 427 & 20.66 & $\mathbf{Q}(\sqrt{-7}, \sqrt{61})$ & $(\mathrm{Y})$ \\
\hline
\end{tabular}




\begin{tabular}{|c|c|c|c|c|c|c|}
\hline$n$ & Type & Generators & $f$ & $r d$ & Simple expression & UC \\
\hline \multirow{31}{*}{4} & \multirow{31}{*}{$\left(2^{*}, 2^{*}\right)$} & $\chi_{3}, \chi_{4}$ & 12 & 3.46 & $\mathbf{Q}\left(\zeta_{12}\right)$ & $\mathrm{Y}$ \\
\hline & & $\chi_{3}, \chi_{7}^{3}$ & 21 & 4.58 & $\mathbf{Q}(\sqrt{-3}, \sqrt{-7})$ & $\mathrm{Y}$ \\
\hline & & $\chi_{3}, \chi_{4} \psi_{8}$ & 24 & 4.89 & $\mathbf{Q}(\sqrt{-3}, \sqrt{-2})$ & $\mathrm{Y}$ \\
\hline & & $\chi_{4}, \chi_{7}^{3}$ & 28 & 5.29 & $\mathbf{Q}(\sqrt{-1}, \sqrt{-7})$ & $\mathrm{Y}$ \\
\hline & & $\chi_{3}, \chi_{11}^{5}$ & 33 & 5.74 & $\mathbf{Q}(\sqrt{-3}, \sqrt{-11})$ & $\bar{Y}$ \\
\hline & & $\chi_{4}, \chi_{11}^{5}$ & 44 & 6.63 & $\mathbf{Q}(\sqrt{-1}, \sqrt{-11})$ & $\mathbf{Y}$ \\
\hline & & $\chi_{7}^{3}, \chi_{4} \psi_{8}$ & 56 & 7.48 & $\mathbf{Q}(\sqrt{-7}, \sqrt{-2})$ & $\bar{Y}$ \\
\hline & & $\chi_{3}, \chi_{19}^{9}$ & 57 & 7.54 & $\mathbf{Q}(\sqrt{-3}, \sqrt{-19})$ & $\bar{Y}$ \\
\hline & & $\chi_{4}, \chi_{19}^{9}$ & 76 & 8.71 & $\mathbf{Q}(\sqrt{-1}, \sqrt{-19})$ & $\mathrm{Y}$ \\
\hline & & $\chi_{7}^{3}, \chi_{11}^{5}$ & 77 & 8.77 & $\mathbf{Q}(\sqrt{-7}, \sqrt{-11})$ & $\mathrm{Y}$ \\
\hline & & $\chi_{4} \psi_{8}, \chi_{11}^{5}$ & 88 & 9.38 & $\mathbf{Q}(\sqrt{-2}, \sqrt{-11})$ & $\mathbf{Y}$ \\
\hline & & $\chi_{3}, \chi_{43}^{21}$ & 129 & 11.35 & $\mathbf{Q}(\sqrt{-3}, \sqrt{-43})$ & $\mathrm{Y}$ \\
\hline & & $\chi_{7}^{3}, \chi_{19}^{9}$ & 133 & 11.53 & $\mathbf{Q}(\sqrt{-7}, \sqrt{-19})$ & $\bar{Y}$ \\
\hline & & $\chi_{4} \psi_{8}, \chi_{19}^{9}$ & 152 & 12.32 & $\mathbf{Q}(\sqrt{-2}, \sqrt{-19})$ & $\bar{Y}$ \\
\hline & & $\chi_{4}, \chi_{43}^{21}$ & 172 & 13.11 & $\mathbf{Q}(\sqrt{-1}, \sqrt{-43})$ & $\bar{Y}$ \\
\hline & & $\chi_{3}, \chi_{67}^{33}$ & 201 & 14.17 & $\mathbf{Q}(\sqrt{-3}, \sqrt{-67})$ & $\bar{Y}$ \\
\hline & & $\chi_{11}^{5}, \chi_{19}^{9}$ & 209 & 14.45 & $\mathbf{Q}(\sqrt{-11}, \sqrt{-19})$ & $\bar{Y}$ \\
\hline & & $\chi_{4}, \chi_{67}^{33}$ & 268 & 16.37 & $\mathbf{Q}(\sqrt{-1}, \sqrt{-67})$ & $\mathrm{Y}$ \\
\hline & & $\chi_{7}^{3}, \chi_{43}^{21}$ & 301 & 17.34 & $\mathbf{Q}(\sqrt{-7}, \sqrt{-43})$ & $\bar{Y}$ \\
\hline & & $\chi_{4} \psi_{8}, \chi_{43}^{21}$ & 344 & 18.54 & $\mathbf{Q}(\sqrt{-2}, \sqrt{-43})$ & $\mathrm{Y}$ \\
\hline & & $\chi_{3}, \chi_{163}^{81}$ & 489 & 22.11 & $\mathbf{Q}(\sqrt{-3}, \sqrt{-163})$ & $(\mathrm{Y})$ \\
\hline & & $\chi_{4} \psi_{8}, \chi_{67}^{33}$ & 536 & 23.15 & $\mathbf{Q}(\sqrt{-2}, \sqrt{-67})$ & $(\mathrm{Y})$ \\
\hline & & $\chi_{4}, \chi_{163}^{81}$ & 652 & 25.53 & $\mathbf{Q}(\sqrt{-1}, \sqrt{-163})$ & $?$ \\
\hline & & $\chi_{11}^{5}, x_{67}^{33}$ & 737 & 27.14 & $\mathbf{Q}(\sqrt{-11}, \sqrt{-67})$ & $?$ \\
\hline & & $\chi_{7}^{3}, \chi_{163}^{81}$ & 1141 & 33.77 & $\mathbf{Q}(\sqrt{-7}, \sqrt{-163})$ & $?$ \\
\hline & & $\chi_{19}^{9}, \chi_{67}^{33}$ & 1273 & 35.67 & $\mathbf{Q}(\sqrt{-19}, \sqrt{-67})$ & $?$ \\
\hline & & $\chi_{11}^{5}, \chi_{163}^{81}$ & 1793 & 42.34 & $\mathbf{Q}(\sqrt{-11}, \sqrt{-163})$ & $?$ \\
\hline & & $\chi_{43}^{21}, \chi_{67}^{33}$ & 2881 & 53.67 & $\mathbf{Q}(\sqrt{-43}, \sqrt{-67})$ & $?$ \\
\hline & & $\chi_{19}^{9}, \chi_{163}^{81}$ & 3097 & 55.65 & $\mathbf{Q}(\sqrt{-19}, \sqrt{-163})$ & $?$ \\
\hline & & $\chi_{43}^{21}, \chi_{163}^{81}$ & 7009 & 83.71 & $Q(\sqrt{-43}, \sqrt{-163})$ & $?$ \\
\hline & & $\chi_{67}^{33}, \chi_{163}^{81}$ & 10921 & 104.50 & $\mathbf{Q}(\sqrt{-67}, \sqrt{-163})$ & $?$ \\
\hline
\end{tabular}




\begin{tabular}{|c|c|c|c|c|c|c|}
\hline$n$ & Type & Generators & $f$ & $r d$ & Simple expression & UC \\
\hline \multirow{17}{*}{6} & \multirow{5}{*}{$6^{*}$} & $\chi_{7}$ & 7 & 5.06 & $\mathbf{Q}\left(\zeta_{7}\right)$ & $\mathrm{Y}$ \\
\hline & & $\chi_{3} \psi_{9}$ & 9 & 5.19 & $\mathbf{Q}\left(\zeta_{9}\right)$ & $Y$ \\
\hline & & $\chi_{19}^{3}$ & 19 & 11.63 & & $Y$ \\
\hline & & $\chi_{43}^{7}$ & 43 & 22.97 & & $(\mathrm{Y})$ \\
\hline & & $\chi_{67}^{11}$ & 67 & 33.24 & & $?$ \\
\hline & \multirow{12}{*}{$\left(2^{*}, 3\right)$} & $\chi_{3}, \chi_{7}^{2}$ & 21 & 6.33 & $\mathbf{Q}(\sqrt{-3}, \cos (2 \pi / 7))$ & $\bar{Y}$ \\
\hline & & $\chi_{4}, \chi_{7}^{2}$ & 28 & 7.31 & $\mathbf{Q}(\sqrt{-1}, \cos (2 \pi / 7))$ & $\mathrm{Y}$ \\
\hline & & $\chi_{4}, \psi_{9}$ & 36 & 8.65 & $\mathbf{Q}(\sqrt{-1}, \cos (2 \pi / 9))$ & $\mathrm{Y}$ \\
\hline & & $\chi_{3}, \chi_{13}^{4}$ & 39 & 9.57 & & $Y$ \\
\hline & & $\chi_{4} \psi_{8}, \chi_{7}^{2}$ & 56 & 10.35 & $\mathbf{Q}(\sqrt{-2}, \cos (2 \pi / 7))$ & $Y$ \\
\hline & & $\chi_{7}^{3}, \psi_{9}$ & 63 & 11.44 & $\mathbf{Q}(\sqrt{-7}, \cos (2 \pi / 9))$ & $\mathbf{Y}$ \\
\hline & & $\chi_{4}, \chi_{19}^{6}$ & 76 & 14.24 & & $Y$ \\
\hline & & $\chi_{11}^{5}, \chi_{7}^{2}$ & 77 & 12.13 & $\mathbf{Q}(\sqrt{-11}, \cos (2 \pi / 7))$ & $\mathrm{Y}$ \\
\hline & & $\chi_{7}^{3}, \chi_{13}^{4}$ & 91 & 14.62 & & $Y$ \\
\hline & & $\chi_{3}, \chi_{31}^{10}$ & 93 & 17.09 & & $\bar{Y}$ \\
\hline & & $\chi_{4} \psi_{8}, \chi_{13}^{4}$ & 104 & 15.63 & & $\mathrm{Y}$ \\
\hline & & $\chi_{3}, \chi_{43}^{14}$ & 129 & 21.25 & & $(\mathrm{Y})$ \\
\hline \multirow{20}{*}{8} & \multirow{2}{*}{$8^{*}$} & $\chi_{4} \psi_{32}$ & 32 & 14.67 & $\mathbf{Q}(i \sin (\pi / 16))$ & $\mathrm{Y}$ \\
\hline & & $\chi_{41}^{5}$ & 41 & 25.77 & & $(\mathrm{Y})$ \\
\hline & \multirow{2}{*}{$\left(2^{*}, 4\right)$} & $\chi_{4}, \psi_{16}$ & 16 & 8.00 & $\mathbf{Q}\left(\zeta_{16}\right)$ & $\mathrm{Y}$ \\
\hline & & $\chi_{3}, \psi_{16}$ & 48 & 11.65 & $\mathbf{Q}(\sqrt{-3}, \cos (\pi / 8))$ & $Y$ \\
\hline & \multirow{10}{*}{$\left(2^{*}, 4^{*}\right)$} & $\chi_{3}, \chi_{5}$ & 15 & 5.79 & $\mathbf{Q}\left(\zeta_{15}\right)$ & $\mathrm{Y}$ \\
\hline & & $\chi_{4}, \chi_{5}$ & 20 & 6.68 & $\mathbf{Q}\left(\zeta_{20}\right)$ & $Y$ \\
\hline & & $\chi_{7}^{3}, \chi_{5}$ & 35 & 8.84 & $\mathbf{Q}\left(\sqrt{-7}, \zeta_{5}\right)$ & $\mathrm{Y}$ \\
\hline & & $\chi_{4} \psi_{8}, \chi_{5}$ & 40 & 9.45 & $\mathbf{Q}\left(\sqrt{-2}, \zeta_{5}\right)$ & $Y$ \\
\hline & & $\chi_{3}, \chi_{4} \psi_{16}$ & 48 & 11.65 & $\mathbf{Q}(\sqrt{-3}, i \sin (\pi / 8))$ & $\mathrm{Y}$ \\
\hline & & $\chi_{4}, \chi_{13}^{3}$ & 52 & 13.69 & & $\mathrm{Y}$ \\
\hline & & $\chi_{7}^{3}, \chi_{13}^{3}$ & 91 & 18.11 & & $\mathrm{Y}$ \\
\hline & & $\chi_{4}, \chi_{37}^{9}$ & 148 & 30.00 & & $?$ \\
\hline & & $\chi_{11}^{5}, \chi_{4} \psi_{16}$ & 176 & 22.31 & $\mathbf{Q}(\sqrt{-11}, i \sin (\pi / 8))$ & $(\mathrm{Y})$ \\
\hline & & $\chi_{4} \psi_{8}, \chi_{29}^{7}$ & 232 & 35.34 & & $?$ \\
\hline & \multirow{6}{*}{$\left(4^{*}, 2\right)$} & $\chi_{5}, \psi_{8}$ & 40 & 9.45 & $\mathbf{Q}\left(\zeta_{5}, \sqrt{2}\right)$ & $\mathrm{Y}$ \\
\hline & & $\chi_{5}, \chi_{13}^{6}$ & 65 & 12.05 & $\mathbf{Q}\left(\zeta_{5}, \sqrt{13}\right)$ & $Y$ \\
\hline & & $\chi_{13}^{3}, \chi_{5}^{2}$ & 65 & 15.30 & & $Y$ \\
\hline & & $\chi_{4} \psi_{16}, \chi_{5}^{2}$ & 80 & 15.04 & $\mathbf{Q}(i \sin (\pi / 8), \sqrt{5})$ & $Y$ \\
\hline & & $\chi_{5}, \chi_{17}^{8}$ & 85 & 13.78 & $\mathbf{Q}\left(\zeta_{5}, \sqrt{17}\right)$ & $\mathrm{Y}$ \\
\hline & & $\chi_{13}^{3}, \psi_{8}$ & 104 & 19.36 & & $\mathrm{Y}$ \\
\hline
\end{tabular}




\begin{tabular}{|c|c|c|c|c|c|c|}
\hline$n$ & Type & Generators & $f$ & $r d$ & Simple expression & $\mathrm{UC}$ \\
\hline \multirow{17}{*}{8} & $\left(2^{*}, 2,2\right)$ & $\chi_{4}, \psi_{8}, \chi_{5}^{2}$ & 40 & 8.94 & $\mathbf{Q}\left(\zeta_{8}, \sqrt{5}\right)$ & $\mathbf{Y}$ \\
\hline & \multirow{10}{*}{$\left(2^{*}, 2,2^{*}\right)$} & $\chi_{4}, \psi_{8}, \chi_{3}$ & 24 & 6.92 & $\mathbf{Q}\left(\zeta_{24}\right)$ & $\bar{Y}$ \\
\hline & & $\chi_{3}, \chi_{5}^{2}, \chi_{4}$ & 60 & 7.74 & $\mathbf{Q}\left(\zeta_{12}, \sqrt{5}\right)$ & $\mathrm{Y}$ \\
\hline & & $\chi_{4}, \psi_{8}, \chi_{11}^{5}$ & 88 & 13.26 & $\mathbf{Q}\left(\zeta_{8}, \sqrt{-11}\right)$ & $\mathbf{Y}$ \\
\hline & & $\chi_{3}, \chi_{5}^{2}, \chi_{7}^{3}$ & 105 & 10.24 & $\mathbf{Q}(\sqrt{-3}, \sqrt{5}, \sqrt{-7})$ & $\mathrm{Y}$ \\
\hline & & $\chi_{3}, \chi_{5}^{2}, \chi_{4} \psi_{8}$ & 120 & 10.95 & $\mathbf{Q}(\sqrt{-3}, \sqrt{5}, \sqrt{-2})$ & $\mathrm{Y}$ \\
\hline & & $\chi_{4}, \chi_{5}^{2} \chi_{7}^{3}$ & 140 & 11.83 & $\mathbf{Q}(\sqrt{-1}, \sqrt{5}, \sqrt{-7})$ & $\mathrm{Y}$ \\
\hline & & $\chi_{3}, \psi_{8}, \chi_{11}^{5}$ & 264 & 16.24 & $\mathbf{Q}(\sqrt{-3}, \sqrt{2}, \sqrt{-11})$ & $Y$ \\
\hline & & $\chi_{7}^{3}, \chi_{5}^{2}, \chi_{4} \psi_{8}$ & 280 & 16.73 & $\mathbf{Q}(\sqrt{-7}, \sqrt{5}, \sqrt{-2})$ & $\mathrm{Y}$ \\
\hline & & $\chi_{4}, \chi_{13}^{6}, \chi_{7}^{3}$ & 364 & 19.07 & $\mathbf{Q}(\sqrt{-1}, \sqrt{13}, \sqrt{-7})$ & $\mathrm{Y}$ \\
\hline & & $\chi_{3}, \chi_{17}^{8}, \chi_{11}^{5}$ & 561 & 23.68 & $\mathbf{Q}(\sqrt{-3}, \sqrt{17}, \sqrt{-11})$ & $(\mathrm{Y})$ \\
\hline & \multirow{6}{*}{$\left(2^{*}, 2^{*}, 2^{*}\right)$} & $\chi_{3}, \chi_{4}, \chi_{7}^{3}$ & 84 & 9.16 & $\mathbf{Q}\left(\zeta_{12}, \sqrt{-7}\right)$ & $\mathrm{Y}$ \\
\hline & & $\chi_{3}, \chi_{4}, \chi_{11}^{5}$ & 132 & 11.48 & $\mathbf{Q}\left(\zeta_{12}, \sqrt{-11}\right)$ & $\bar{Y}$ \\
\hline & & $\chi_{3}, \chi_{7}^{3}, \chi_{4} \psi_{8}$ & 168 & 12.96 & $\mathbf{Q}(\sqrt{-3}, \sqrt{-7}, \sqrt{-2})$ & $\mathbf{Y}$ \\
\hline & & $\chi_{3}, \chi_{4}, \chi_{19}^{9}$ & 228 & 15.09 & $\mathbf{Q}\left(\zeta_{12}, \sqrt{-19}\right)$ & $\mathbf{Y}$ \\
\hline & & $\chi_{4}, \chi_{7}^{3}, \chi_{19}^{9}$ & 532 & 23.06 & $\mathbf{Q}(\sqrt{-1}, \sqrt{-7}, \sqrt{-19})$ & $(\mathbf{Y})$ \\
\hline & & $\chi_{3}, \chi_{11}^{5}, \chi_{19}^{9}$ & 627 & 25.03 & $\mathbf{Q}(\sqrt{-3}, \sqrt{-11}, \sqrt{-19})$ & $(\mathrm{Y})$ \\
\hline \multirow{3}{*}{10} & $10^{*}$ & $\chi_{11}$ & 11 & 8.65 & $\mathbf{Q}\left(\zeta_{11}\right)$ & $\mathbf{Y}$ \\
\hline & \multirow{2}{*}{$\left(2^{*}, 5\right)$} & $\chi_{3}, \chi_{11}^{2}$ & 33 & 11.79 & $\mathbf{Q}(\sqrt{-3}, \cos (2 \pi / 11))$ & $\mathbf{Y}$ \\
\hline & & $\chi_{4}, \chi_{11}^{2}$ & 44 & 13.61 & $\mathbf{Q}(\sqrt{-1}, \cos (2 \pi / 11))$ & $\mathrm{Y}$ \\
\hline \multirow{18}{*}{12} & \multirow{3}{*}{$12^{*}$} & $\chi_{13}$ & 13 & 10.49 & $\mathbf{Q}\left(\zeta_{13}\right)$ & $\mathrm{Y}$ \\
\hline & & $\chi_{37}^{3}$ & 37 & 27.38 & & $(\mathrm{Y})$ \\
\hline & & $\chi_{61}^{5}$ & 61 & 43.30 & & $?$ \\
\hline & \multirow{8}{*}{$\left(2^{*}, 6^{*}\right)$} & $\chi_{3}, \chi_{7}$ & 21 & 8.76 & $\mathbf{Q}\left(\zeta_{21}\right)$ & $\mathrm{Y}$ \\
\hline & & $\chi_{4}, \chi_{7}$ & 28 & 10.12 & $\mathbf{Q}\left(\zeta_{28}\right)$ & $\mathbf{Y}$ \\
\hline & & $\chi_{4}, \chi_{3} \psi_{9}$ & 36 & 10.39 & $\mathbf{Q}\left(\zeta_{36}\right)$ & $\mathrm{Y}$ \\
\hline & & $\chi_{4} \psi_{8}, \chi_{7}$ & 56 & 14.31 & $\mathbf{Q}\left(\sqrt{-2}, \zeta_{7}\right)$ & $\mathrm{Y}$ \\
\hline & & $\chi_{7}^{3}, \chi_{3} \psi_{9}$ & 63 & 13.74 & $\mathbf{Q}\left(\sqrt{-7}, \zeta_{9}\right)$ & $\mathrm{Y}$ \\
\hline & & $\chi_{4}, \chi_{19}^{3}$ & 76 & 23.26 & & $(\mathrm{Y})$ \\
\hline & & $\chi_{11}^{5}, \chi_{7}$ & 77 & 16.78 & $\mathbf{Q}\left(\sqrt{-11}, \zeta_{7}\right)$ & $\mathbf{Y}$ \\
\hline & & $\chi_{3}, \chi_{43}^{7}$ & 129 & 39.79 & & $?$ \\
\hline & \multirow{2}{*}{$\left(4^{*}, 3\right)$} & $\chi_{5}, \chi_{7}^{2}$ & 35 & 12.23 & $\mathbf{Q}\left(\zeta_{5}, \cos (2 \pi / 7)\right)$ & $\mathbf{Y}$ \\
\hline & & $\chi_{5}, \psi_{9}$ & 45 & 14.46 & $\mathbf{Q}\left(\zeta_{5}, \cos (2 \pi / 9)\right)$ & $\mathrm{Y}$ \\
\hline & \multirow{3}{*}{$\left(6^{*}, 2\right)$} & $\chi_{7}, \chi_{5}^{2}$ & 35 & 11.31 & $\mathbf{Q}\left(\zeta_{7}, \sqrt{5}\right)$ & $\mathbf{Y}$ \\
\hline & & $\chi_{3} \psi_{9}, \chi_{5}^{2}$ & 45 & 11.61 & $\mathbf{Q}\left(\zeta_{9}, \sqrt{5}\right)$ & $\mathrm{Y}$ \\
\hline & & $\chi_{3} \psi_{9}, \psi_{8}$ & 72 & 14.69 & $\mathbf{Q}\left(\zeta_{9}, \sqrt{2}\right)$ & $\mathrm{Y}$ \\
\hline & \multirow{2}{*}{$\left(2^{*}, 2,3\right)$} & $\chi_{4}, \psi_{8}, \chi_{7}^{2}$ & 56 & 14.63 & $\mathbf{Q}\left(\zeta_{8}, \cos (2 \pi / 7)\right)$ & $\mathrm{Y}$ \\
\hline & & $\chi_{3}, \chi_{5}^{2}, \chi_{7}^{2}$ & 105 & 14.17 & $\mathbf{Q}(\sqrt{-3}, \sqrt{5}, \cos (2 \pi / 7))$ & $\mathrm{Y}$ \\
\hline
\end{tabular}




\begin{tabular}{|c|c|c|c|c|c|c|}
\hline$n$ & Type & Generators & $f$ & $r d$ & Simple expression & UC \\
\hline \multirow{8}{*}{12} & \multirow{8}{*}{$\left(2^{*}, 2^{*}, 3\right)$} & $\chi_{3}, \chi_{4}, \chi_{7}^{2}$ & 84 & 12.67 & $\mathbf{Q}\left(\zeta_{12}, \cos (2 \pi / 7)\right)$ & $\bar{Y}$ \\
\hline & & $\chi_{3}, \chi_{4} \psi_{8}, \chi_{7}^{2}$ & 168 & 17.92 & $\mathbf{Q}(\sqrt{-3}, \sqrt{-2}, \cos (2 \pi / 7))$ & $Y$ \\
\hline & & $\chi_{3}, \chi_{11}^{5}, \chi_{7}^{2}$ & 231 & 21.02 & $\mathbf{Q}(\sqrt{-3}, \sqrt{-11}, \cos (2 \pi / 7))$ & $(\mathrm{Y})$ \\
\hline & & $\chi_{4}, \chi_{7}^{3}, \psi_{9}$ & 252 & 22.89 & $\mathbf{Q}\left(\zeta_{12}, \cos (2 \pi / 9)\right)$ & $(\mathrm{Y})$ \\
\hline & & $\chi_{3}, \chi_{7}^{3}, \chi_{13}^{4}$ & 273 & 25.33 & & $(\mathrm{Y})$ \\
\hline & & $\chi_{4}, \chi_{11}^{5}, \chi_{7}^{2}$ & 308 & 24.27 & $\mathbf{Q}(\sqrt{-1}, \sqrt{-11}, \cos (2 \pi / 7))$ & $(\mathrm{Y})$ \\
\hline & & $\chi_{3}, \chi_{4} \psi_{8}, \chi_{13}^{4}$ & 312 & 27.08 & & $(\mathrm{Y})$ \\
\hline & & $\chi_{7}^{3}, \chi_{4} \psi_{8}, \chi_{13}^{4}$ & 728 & 41.37 & & $?$ \\
\hline \multirow{2}{*}{14} & \multirow{2}{*}{$14^{*}$} & $\chi_{43}^{3}$ & 43 & 32.86 & & $?$ \\
\hline & & $\chi_{7}^{3} \psi_{49}$ & 49 & 32.29 & & $?$ \\
\hline \multirow{12}{*}{16} & $16^{*}$ & $\chi_{17}$ & 17 & 14.24 & $\mathbf{Q}\left(\zeta_{17}\right)$ & $\bar{Y}$ \\
\hline & $\left(2^{*}, 8\right)$ & $\chi_{4}, \psi_{32}$ & 32 & 16.00 & $\mathbf{Q}\left(\zeta_{32}\right)$ & $\bar{Y}$ \\
\hline & $\left(2^{*}, 8^{*}\right)$ & $\chi_{3}, \chi_{4} \psi_{32}$ & 96 & 25.41 & $\mathbf{Q}(\sqrt{-3}, i \sin (\pi / 16))$ & $(\mathrm{Y})$ \\
\hline & \multirow{2}{*}{$\left(4^{*}, 4^{*}\right)$} & $\chi_{5}, \chi_{13}^{3}$ & 65 & 22.89 & & $(\mathrm{Y})$ \\
\hline & & $\chi_{5}, \chi_{4} \psi_{16}$ & 80 & 22.49 & $\mathbf{Q}\left(\zeta_{5}, i \sin (\pi / 8)\right)$ & $(\mathrm{Y})$ \\
\hline & $\left(2^{*}, 2,4^{*}\right)$ & $\chi_{4}, \psi_{8}, \chi_{5}$ & 40 & 13.37 & $\mathbf{Q}\left(\zeta_{40}\right)$ & $Y$ \\
\hline & \multirow{4}{*}{$\left(2^{*}, 2^{*}, 4^{*}\right)$} & $\chi_{3}, \chi_{4}, \chi_{5}$ & 60 & 11.58 & $\mathbf{Q}\left(\zeta_{60}\right)$ & $\bar{Y}$ \\
\hline & & $\chi_{3}, \chi_{7}^{3}, \chi_{5}$ & 105 & 15.32 & $\mathbf{Q}\left(\zeta_{15}, \sqrt{-7}\right)$ & $\bar{Y}$ \\
\hline & & $\chi_{3}, \chi_{4} \psi_{8}, \chi_{5}$ & 120 & 16.38 & $\mathbf{Q}\left(\zeta_{15}, \sqrt{-2}\right)$ & $\mathbf{Y}$ \\
\hline & & $\chi_{4}, \chi_{7}^{3}, \chi_{5}$ & 140 & 17.69 & $\mathbf{Q}\left(\zeta_{20}, \sqrt{-7}\right)$ & $\bar{Y}$ \\
\hline & $\left(2^{*}, 4,2\right)$ & $\chi_{4}, \psi_{16}, \chi_{5}^{2}$ & 80 & 17.88 & $\mathbf{Q}\left(\zeta_{16}, \sqrt{5}\right)$ & $\bar{Y}$ \\
\hline & $\left(2^{*}, 4,2^{*}\right)$ & $\chi_{4}, \psi_{16}, \chi_{3}$ & 48 & 13.85 & $\mathbf{Q}\left(\zeta_{48}\right)$ & $\bar{Y}$ \\
\hline \multirow{3}{*}{18} & \multirow{2}{*}{$18^{*}$} & $\chi_{19}$ & 19 & 16.13 & $\mathbf{Q}\left(\zeta_{19}\right)$ & $\bar{Y}$ \\
\hline & & $\chi_{3}, \psi_{27}$ & 27 & 15.58 & $\mathbf{Q}\left(\zeta_{27}\right)$ & $\bar{Y}$ \\
\hline & $\left(6^{*}, 3\right)$ & $\chi_{3} \psi_{9}, \chi_{7}^{2}$ & 63 & 19.01 & $\mathbf{Q}\left(\zeta_{9}, \cos (2 \pi / 7)\right)$ & $\bar{Y}$ \\
\hline \multirow{4}{*}{20} & $20^{*}$ & $\chi_{5} \psi_{25}$ & 25 & 16.71 & $\mathbf{Q}\left(\zeta_{25}\right)$ & $\mathrm{Y}$ \\
\hline & \multirow{2}{*}{$\left(2^{*}, 10^{*}\right)$} & $\chi_{3}, \chi_{11}$ & 33 & 14.99 & $\mathbf{Q}\left(\zeta_{33}\right)$ & $\bar{Y}$ \\
\hline & & $\chi_{4}, \chi_{11}$ & 44 & 17.30 & $\mathbf{Q}\left(\zeta_{44}\right)$ & $\bar{Y}$ \\
\hline & $\left(2^{*}, 2^{*}, 5\right)$ & $\chi_{3}, \chi_{4}, \chi_{11}^{2}$ & 132 & 23.58 & $\mathbf{Q}\left(\zeta_{12}, \cos (2 \pi / 11)\right)$ & $(\mathrm{Y})$ \\
\hline \multirow{5}{*}{24} & \multirow{2}{*}{$\left(4^{*}, 6^{*}\right)$} & $\chi_{5}, \chi_{7}$ & 35 & 16.92 & $\mathbf{Q}\left(\zeta_{35}\right)$ & $\bar{Y}$ \\
\hline & & $\chi_{5}, \chi_{3} \psi_{9}$ & 45 & 17.37 & $\mathbf{Q}\left(\zeta_{45}\right)$ & $\bar{Y}$ \\
\hline & $\left(2^{*}, 2,6^{*}\right)$ & $\chi_{3}, \chi_{5}^{2}, \chi_{7}$ & 105 & 19.60 & $\mathbf{Q}\left(\zeta_{21}, \sqrt{5}\right)$ & $\bar{Y}$ \\
\hline & $\left(2^{*}, 2^{*}, 6^{*}\right)$ & $\chi_{3}, \chi_{4}, \chi_{7}$ & 84 & 17.53 & $\mathbf{Q}\left(\zeta_{84}\right)$ & $\bar{Y}$ \\
\hline & $\left(2^{*}, 3,4^{*}\right)$ & $\chi_{3}, \chi_{7}^{2}, \chi_{5}$ & 105 & 21.19 & $\mathbf{Q}\left(\zeta_{15}, \cos (2 \pi / 7)\right)$ & $\bar{Y}$ \\
\hline
\end{tabular}




\section{BIBLIOGRAPHY}

1. F. Diaz y Diaz, Tables minorant la racine n-ieme du discriminant d'un corps de degré $n$, Publications Mathématiques d'Orsay 80, 6, Université de Paris-Sud, Département de Mathématique, Orsay, 59pp.

2. S. Chowla, Note on Dirichlet's L-functions, Acta Arith. 1 (1936), 113-114.

3. S. Chowla, M. J. DeLeon, and P. Hartung, On a hypothesis implying the non-vanishing of Dirichlet's L-series $L(s, \chi)>0$ for $s>0$ and real odd characters $\chi$, J. Reine Angew. Math. 262/263 (1973), 415-419.

4. S. Chowla and M. J. DeLeon, A note on the Hecke hypothesis and the determination of imaginary quadratic fields with class number 1, J. Number Theory 6 (1974), 261-263.

5. S. Chowla and P. Hartung, $A$ note on the hypothesis that $L(s, \chi)>0$ for all real non-principal characters $\chi$ and all $s>0$, J. Number Theory 6 (1974), 271-275.

6. H. Hasse, Über die Klassenzahl abelscher Zahlkörper, Akademie-Verlag, Berlin, 1952.

7. H. Heilbronn, On real characters, Acta Arith. 2 (1937), 212-213.

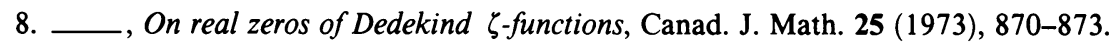

9. L. K. Hua, On the least solution of Pell's equation, Bull. Amer. Math. Soc. 48 (1942), 731-735.

10. S. Kuroda, On a theorem of Minkowski, Sugaku 14 (1962/63), 171-172. (Japanese)

11. F. van der Linden, Class number computations of real abelian number fields, Math. Comp. 39 (1982), 693-707.

12. S. Louboutin, Minoration au point 1 des fonctions $L$ et détermination des corps sextiques abéliens totalement imaginaires principaux, Acta Arith. 62 (1992), 109-124.

13. __ Lower bounds for relative class numbers of CM-fields, Proc. Amer. Math. Soc. 120 (1994), 425-434.

14. __ Zéros réels des fonctions zeta et minoration de nombres de classes. Application à la majoration des discriminants de certains types des corps nombres, to appear in Séminaire de Thèorie des Nombres, Paris 1991/92 Progress in Math., Birkhäuser.

15. M. E. Low, Real zeros of the Dedekind zeta function of an imaginary quadratic field, Acta Arith. 14 (1968), 117-140.

16. S. Mäki, The determination of units in real cyclic sextic fields, Lecture Notes in Math., vol. 797, Springer-Verlag, Berlin and New York, 1980.

17. J. Martinet, Tours de corps de classes et discriminants, Invent. Math. 44 (1978), 65-73.

18. __ Petit discriminant des corps de nombres, Number theory days, 1980 (Exeter, 1980) London Math. Soc. Lecture Note Ser. 56, Cambridge Univ. Press, Cambridge, New York, 1982, pp. 151-193.

19. J. M. Masley, Odlyzko bounds and class number problems, Algebraic Number Fields: $L$ functions and Galois Properties (A. Fröhlich, ed.), (Proc. Sympos. Univ., Durham, 1975), Academic Press, London, 1977, pp. 465-474.

20. _ Class numbers of real cyclic number fields with small conductor, Compositio Math. 37 (1978), 297-319.

21. J. M. Masley and H. L. Montgomery, Cyclotomic fields with unique factorization, J. Reine Angew. Math. 286/287 (1976), 248-256.

22. C. Moser et J. J. Payan, Majoration du nombre de classes d'un corps cubique cyclique de conducteur premier, J. Math. Soc. Japan 33 (1981), 701-706.

23. T. Nakahara, Examples of algebraic number fields which have not unramified extensions, Rep. Fac. Sci. Engrg. Saga Univ. Math. 1 (1973), 61-68.

24. A. M. Odlyzko, Discriminant bounds, (unpublished tables), Nov. 29th, 1976.

25. G. Schrutka von Rechtenstamm, Tabelle der (relativ-)Klassenzahlen der Kreiskörper, deren $\phi$-Funktion des Wurzelexponenten (Grad) nicht grösser als 256 ist, Abh. Deutschen Akad. Wiss. Berlin, Kl. Math. Phys. 2 (1964), 1-64.

26. J. B. Rosser, Real zeros of Dirichlet L-series, J. Res. Nat. Bur. Standards Sect. B 45 (1950), 505-514. 
27. H. M. Stark, Some effective versions of the Brauer-Siegel theorem, Invent. Math. 23 (1974), 135-152.

28. K. Uchida, Class numbers of imaginary abelian number fields, I, Tôhoku Math. J. 23 (1971), 97-104.

29. __ Imaginary abelian number fields with class number one, Tôhoku Math. J. 24 (1972), 487-499.

30. _ Imaginary abelian number fields of degree $2^{m}$ with class number one (Correction in Zbl. Math. 612(1987) o12011), Class Numbers and Fundamental Units of Algebraic Number Fields, Proc. Internat. Conf. Katata/Jap., 1986, pp. 151-170.

31. L. C. Washington, Introduction to cyclotomic fields, Graduate Texts in Math., vol. 83, Springer-Verlag, Berlin and New York, 1980.

32. K. Yamamura, On unramified Galois extensions of real quadratic number fields, Osaka J. Math. 23 (1986), 471-486.

33. $\ldots$ Examples of polynomials $X^{n}+a_{1} X^{n-1}+\cdots+a_{n}$ giving an unramified $A_{n}$-extension of a real quadratic number field $\mathbf{Q}(\sqrt{D})$ with class number one (unpublished tables).

34. number fields, J. Fac. Sci. Univ. Tokyo Sect. IA 38 (1991), 99-135.

Department of Mathematics, National Defence Academy, Hashirimizu Yokosuka 239, JAPAN

E-mail address: yamamura@jpnnda.bitnet 